\title{
Dos nuevas especies de Miconia (Melastomataceae) del piedemonte oriental de la cordillera Central de Antioquia, Colombia
}

Two new species of Miconia (Melastomataceae) from the eastern foothills of the Cordillera Central of Antioquia, Colombia

\section{Humberto Mendoza-Cifuentes, Julián Aguirre-Santoro y Álvaro Idárraga}

\section{Resumen}

Se describen dos especies de Melastomataceae de frutos carnosos procedentes del departamento de Antioquia, Colombia: Miconia mailynii y Miconia hernandogarciae. Miconia mailynii se caracteriza por la presencia de mirmecófilos, hojas levemente anisófilas e inflorescencias terminales; los taxones más cercanos corresponden a especies del género Clidemia de Panamá y Costa Rica. Miconia hernandogarciae se asocia al clado Octopleura y se distingue por poseer inflorescencias terminales y hojas con venación basal. Estas dos especies se consideran endémicas al piedemonte asociado al Magdalena Medio de las cordilleras Central y Oriental de Colombia y, debido a su distribución restringida y especificidad de hábitat, se recomienda su categorización como En peligro (EN).

Palabras clave. Bosques subandinos. Flora de Colombia. Miconieae. Norte de los Andes. Taxonomía.

\begin{abstract}
Miconia mailynii and Miconia hernandogarciae, two new species of berry-fruited Melastomataceae from the department of Antioquia, Colombia, are described. Miconia mailynii is characterized by the presence of ant domatia, slightly anisophyllous leaves, and terminal inflorescences. This species is probably related to those species previously placed in the genus Clidemia from Panama and Costa Rica. Miconia hernandogarciae is associated within the clade Octopleura, and can be distinguished by its terminal inflorescences and leaves with basal venation. These two species are endemic to the foothills of the Cordillera Central and Cordillera Oriental around the central Magdalena Valley of Colombia. Because of their restricted distribution and habitat specificity, the categorization of species these species as Endangered $(\mathrm{EN})$ is recommended.
\end{abstract}

Keywords. Flora of Colombia. Miconieae. Northern Andes. Subandean Forest. Taxonomy.

\section{Introducción}

Las actuales condiciones generadas por el proceso de paz colombiano han permitido visitar y explorar regiones de gran interés biológico del territorio nacional, antes inaccesibles por un largo conflicto armado interno. Durante una reciente expedición a una de estas zonas, en los bosques húmedos 
del piedemonte aledaño al Magdalena Medio de la vertiente oriental de la cordillera Central (departamento de Antioquia), se encontraron dos nuevas especies de Melastomataceae.

Colombia es el segundo país con mayor diversidad de la familia Melastomataceae en el planeta, con 990 especies, un tercio de ellas endémicas (Almeda et al., 2016). Dentro de esta familia, el género mejor representado en este país es Miconia Ruiz \& Pav., del cual se reconocen 382 especies (Almeda et al., 2016). En Colombia, Miconia es diverso en bosques de tierras bajas y andinos, en especial en áreas muy húmedas como el Chocó Biogeográfico, la Amazonia, el Magdalena Medio y el piedemonte de los Andes.

Miconia, que fue recientemente recircunscrito con base en análisis filogenéticos (Michelangeli et al., 2016), reúne a todas las especies de Melastomataceae con frutos carnosos, flores pequeñas e inflorescencias multifloras, previamente agrupadas en la tribu Miconiae, un grupo representado por 18-20 géneros de distribución exclusivamente neotropical. Debido a que no todas las especies de estos géneros han sido formalmente transferidas a Miconia, se calcula que la verdadera riqueza del género en Colombia está alrededor de las 600 especies. Actualmente, las nuevas especies de la tribu están siendo descritas en Miconia (p. ej. Ionta et al., 2012; Michelangeli y Meier, 2013; Gamba y Almeda, 2014).

Acogiendo la nueva circunscripción de Miconia, en el presente trabajo se describen e ilustran dos nuevas especies provenientes de los bosques húmedos del municipio de Carmen de Viboral en Antioquia, Colombia. La primera especie corresponde a un sufrútice con mirmecodomacios en las hojas e inflorescencias terminales, la cual se asocia a especies de Panamá y Costa Rica del género Clidemia. La segunda es una especie de inflorescencias terminales, pétalos agudos $\mathrm{y}$ frutos costillados, que se asocia al clado Octopleura de Miconia, un grupo recientemente revisado por Gamba y Almeda (2014). Además de las descripciones de estas nuevas especies, el presente trabajo también incluye ilustraciones, distribuciones geográficas y notas sobre su estado de conservación.

\section{Materiales y métodos}

Para confirmar la identidad y afinidades taxonómicas de las nuevas especies se revisó literatura pertinente al género Miconia clado Octopleura y el género Clidemia, la base de datos en línea del Herbario Nacional Colombiano (COL) (http://www.biovirtual.unal.edu.co/es/ colecciones/search/plants/), el catálogo de Melastomataceae de Colombia (Almeda et al., 2016) y el tratamiento taxonómico de la flora Mesoamericana (Almeda, 2009). Una vez identificadas las novedades taxonómicas, se procedió a confrontar directamente las colecciones del presente hallazgo con colecciones de los herbarios Nacional Colombiano (COL), Federico Medem Bogotá (FMB) y Universidad de Antioquia (HUA), en donde se identificaron otras colecciones correspondientes a los nuevos taxones. Adicionalmente, se consultaron las imágenes tipo de la totalidad de las especies de Miconia clado Octopleura y Clidemia de Colombia usando la base de datos de Global Plants JSTOR (http://plants. jstor.org/search?plant).

Para elaborar las descripciones se realizaron medidas de las partes vegetativas y de la inflorescencia en material seco de herbario y utilizando un calibrador digital de 0,01 $\mathrm{mm}$ de precisión. Las medidas de las partes florales se basaron en flores frescas preservadas en alcohol al $70 \%$ y procedentes de las plantas que fueron designadas como tipos. Se realizaron medidas de las partes florales y tricomas en un microscopio Leica S8AP0 y las imágenes fueron tomadas con una cámara MC190 HD. Los tipos de tricomas se identifican con base en el Atlas de tricomas de Melastomataceae de Wurdack (1986). Finalmente, se evalúo el estado de conservación de los dos taxones con base en los criterios de amenaza de la $\operatorname{IUCN}(2001,2010)$. 


\section{Resultados}

\section{Miconia mailynii Humberto Mend. \& Aguirre-Santoro, sp. nov. (Figuras 1, 2 y 3).}

This species is recognized by its slightly anisophyllous leaves, both leaves of each node with ant domatia at the petiole apex, basinerved leaf, base of the leaf blade cordate, terminal paniculate inflorescences, and anthers connective slightly bilobed at the base with glandular trichomes. This species is related to Clidemia taurina Gleason (1950:360) but the latter is different in its axillar congested inflorescence and strongly reflexed trichomes on the stem. The species is also related to Clidemia lanuginosa Almeda (2004:109) but the latter is different in its isophyllous leaf, ant domatia at the base of the leaf blade, leaf plinervate, base of the leaf blade rounded, and anther connective glabrous.

TIPO: COLOMBIA. Antioquia. Municipio de Carmen de Viboral, vereda El Porvenir, camino a lo largo de filo de la montaña que conduce entre las fincas La Ilusión y La Troja, $05^{\circ} 53^{\prime} 21.6^{\prime \prime} \mathrm{N}-75^{\circ} 10^{\prime} 25,9^{\prime \prime} \mathrm{O}, 1447 \mathrm{~m}, 03-X \mathrm{II}-2016$ (fl, fr), H. Mendoza et al. 19547 (holotipo: FMB 112363!; isotipos: FMB 112364!, HUA!, COL!, CUVC!, CAUP!).

Arbustos poco ramificados de 2,5-4 m de altura. Ramas teretes; entrenudos $5-9 \mathrm{~cm}$ de largo. Entrenudos, pecíolo e inflorescencia densamente villosos, con tricomas subulados lisos tipo 11 (sensu Wurdack, 1986), erectos, 3,5-4,5 mm de largo; también presentan en menor densidad diminutos tricomas glandulares alargados tipo 2 (sensu Wurdack, 1986), 0,1-0,15 mm de largo. Hojas pecioladas, dimorfas, desiguales en tamaño en el mismo nudo, ambas hojas de un nudo con domacios en el ápice del pecíolo. Domacios oblongos a ovados a manera de vejiga, densamente villosos, con tricomas similares a los del pecíolo; en hojas mayores 9-10,5 × 6-10 mm; en hojas menores 6-9 × 4-7 mm. Hoja mayor con pecíolo 1,2-2,7 cm de largo incluyendo el domacio; lámina oblonga a oblongo-obovada, 20-27 × 10$13 \mathrm{~cm}$; ápice obtuso a redondeado, acuminado; acumen 5,2-20,1 mm de largo; base cordada, margen dentado-ciliolada; haz pubescente, tricomas subulados lisos (tipo 11) de 2,8-4,3 mm de largo; envés pubescente sobre todas la superficie, con tricomas similares a los de la haz. Hoja menor con lámina oblonga a oblongo-ovada; ápice obtuso a redondeado, acuminado; acumen 3,5$5 \mathrm{~mm}$ de largo; base cordada; margen dentadociliolada; haz y envés igual de pubescente que las hojas mayores. Venación en hojas mayores con 3-4 pares de venas secundarias basales acompañando a la vena media, solo dos llegan al ápice; en hojas menores con 3 pares de venas secundarias. Inflorescencia terminal, paniculada, $6,5-25 \mathrm{~cm}$ de largo, sésil o pedunculada, con más de 70 flores; pedúnculo 3,5-7 cm de largo; eje central con 4-5 nudos de ramificación; paracladios basales 2-9 cm de largo; ápice de las ramas trifloros; con o sin brácteas en el nudo basal a manera de pequeñas hojas, con lámina ovada $10 \times 8 \mathrm{~mm}$; brácteas de nudos superiores y secundarios filamentosas, 3-12 × 0,3-0,5 mm. Flores 5-meras, diplostemonas, pediceladas; botón floral 6-7 $\mathrm{mm}$ de largo; pedicelo 0,6-1 mm de largo. Hipanto cilíndrico; parte fusionada al ovario 0,9-1,2 $\mathrm{mm}$ de largo; tálamo libre del ovario 1,6-1,8 mm de largo; externamente con tricomas subulados tipo 11, 1,6-3 mm de largo; torus laminar, formando un anillo de 0,2-0,3 mm de alto. Cáliz lobulado, externamente con largos tricomas subulados similares al hipanto; tálamo 1,03-1,07 mm de largo; lóbulos anchamente triangulares, 0,3-0,4 $\mathrm{mm}$ de largo y 1,3-1,4 mm de ancho; diente dorsal linear, 1,3-2 mm de largo, con tricomas subulados de 1,9-2,5 mm de largo. Corola patente; pétalos obovados, 3-4 × 2-2,6 $\mathrm{mm}$, apicalmente redondeados, blancos, glabros. Estambres levemente dimorfos, los opositisépalos mayores por la antera levemente más larga y robusta. Estambres opositipétalos con filamento 1,5-1,6 mm de largo, glabro; antera 2-2,96 × 0,4-0,5 $\mathrm{mm}$, subulada, con un poro oblongo ventralmente inclinado de 0,3-0,4 $\mathrm{mm}$ en la parte más ancha; conectivo prolongado en la base $0,2-0,3 \mathrm{~mm}$, bilobado, lóbulos 0,16-0,18 × 0,2-0,22 mm, con tricomas glandulares estipitados (tipo 1) 0,140,24 mm de largo. Estambres opositisépalos con 
filamento 1,8-2,2 mm de largo, glabro; antera 3-3,6 $\times$ 0,53-0,55 mm; conectivo levemente prologado en la base 0,2-0,3 mm, bilobado, lóbulos de similar tamaño o un poco más pequeños que en el verticilo opositipétalo, con tricomas glandulares similares a la serie opositipétalos. Ovario 3 locular, $1 / 2$ ínfero, 1,5-1,6 × 1,2-1,4 mm; ápice 0,5-0,6 mm de largo, redondeado-estriado, coronado con ralos tricomas diminutos; placentas con rudimentos solo en la superficie dorsal. Estilo cilíndrico, 4,5-4,6 × 0,5-0,6 $\mathrm{mm}$, glabro; estigma puntiforme, $0,44-0,55 \mathrm{~mm}$ de diámetro. Fruto baya, globoso, $5 \mathrm{~mm}$ de diámetro. Semillas cuneiformes con lados aristados, 0,5-1,2 $\mathrm{mm}$ de largo; testa minutamente tuberculada, de color crema; área de la rafe cóncava, del mismo color de la testa o levemente más oscura.

Fenología. Recolectada con flores en octubre y diciembre.

Etimología. El epíteto específico es en honor a la bióloga Mailyn González, coordinadora del proyecto Colombia $\mathrm{BIO}$ en el Instituto de Investigación de Recursos Biológicos Alexander von Humboldt.

Distribución y hábitat. Conocida de las colecciones tipo en Antioquia y una segunda localidad en el departamento de Cundinamarca. Posiblemente se encuentre en los flancos de las cordilleras Central y Oriental que colindan con el Magdalena Medio, en altitudes entre los 1400 y 1500 m (Figura 4). La vegetación en esta altitud es catalogada como bosque subandino de acuerdo con van der Hammen y Rangel-Ch. (1997). El tipo fue recolectado en zonas de bosque en buen estado de conservación.

Estado de conservación. Miconia mailynii se conoce solo de dos localidades asociadas a los piedemontes que colindan con el Magdalena Medio, crece en una franja altitudinal estrecha, está asociada a zonas de bosque y es poco frecuente en la zona de la localidad tipo. Por estas condiciones cumple el criterio B1biii de la UICN, por lo que se recomienda catalogarla como especie En peligro (EN).

Paratipos: COLOMBIA. Antioquia. Carmen de Viboral, vereda El Porvenir, camino a lo largo del filo de la montaña que conduce entre las fincas La Ilusión y La Troja, $05^{\circ} 53^{\prime} 28.3^{\prime \prime} \mathrm{N}-75^{\circ} 10^{\prime} 27,5^{\prime \prime} \mathrm{O}$, 1330 m, 03-XII-2016 (fl), H. Mendoza et al. 19545 (FMB 112365!, HUA!). Cundinamarca. Yacopí, Inspección de policía de Guadualito, vereda La Laguna, 1465 m, 27-X-1995 (fl), M. E. Morales et al. 460 (COL 384124!, FMB 70467!).

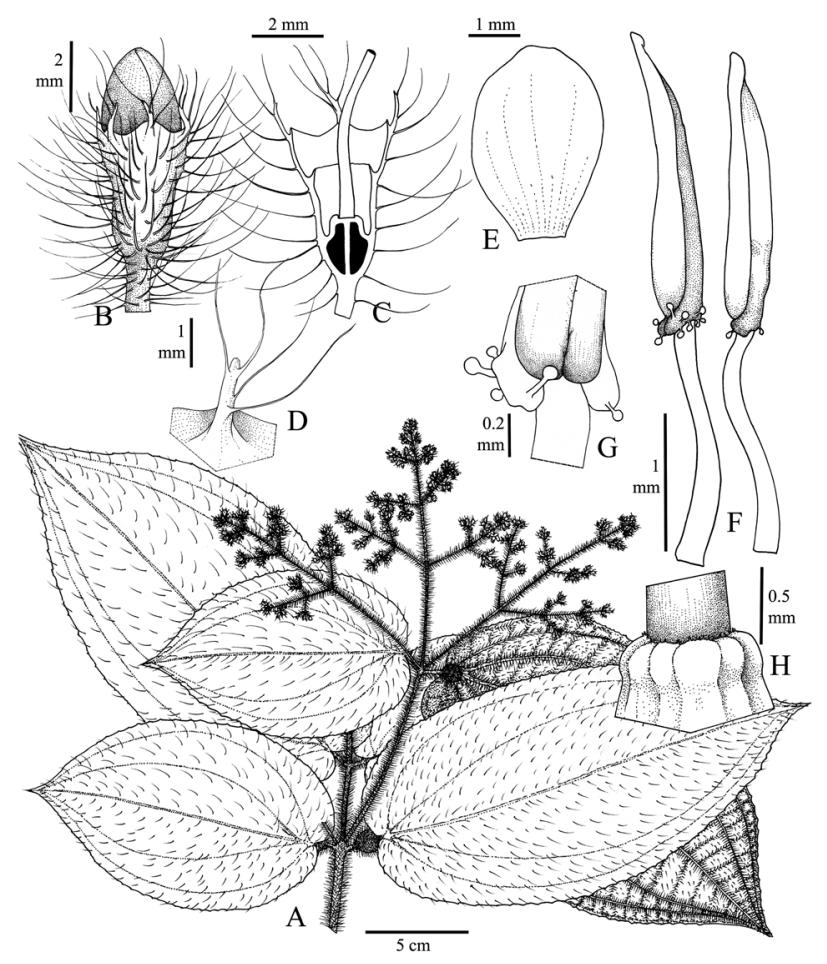

Figura 1. Miconia mailynii. A. Rama florífera. B. Botón floral. C. Corte longitudinal del hipantoovario y del estilo. D. Diente dorsal del cáliz. E. Pétalo. F. Estambres (derecha opositipétalo, izquierda opositisépalo). G. Detalle de la base de la antera en vista ventral del estambre opositipétalo. H. Ápice del ovario. Basado en H. Mendoza et al. 19457 (FMB). Ilustración: Humberto Mendoza. 

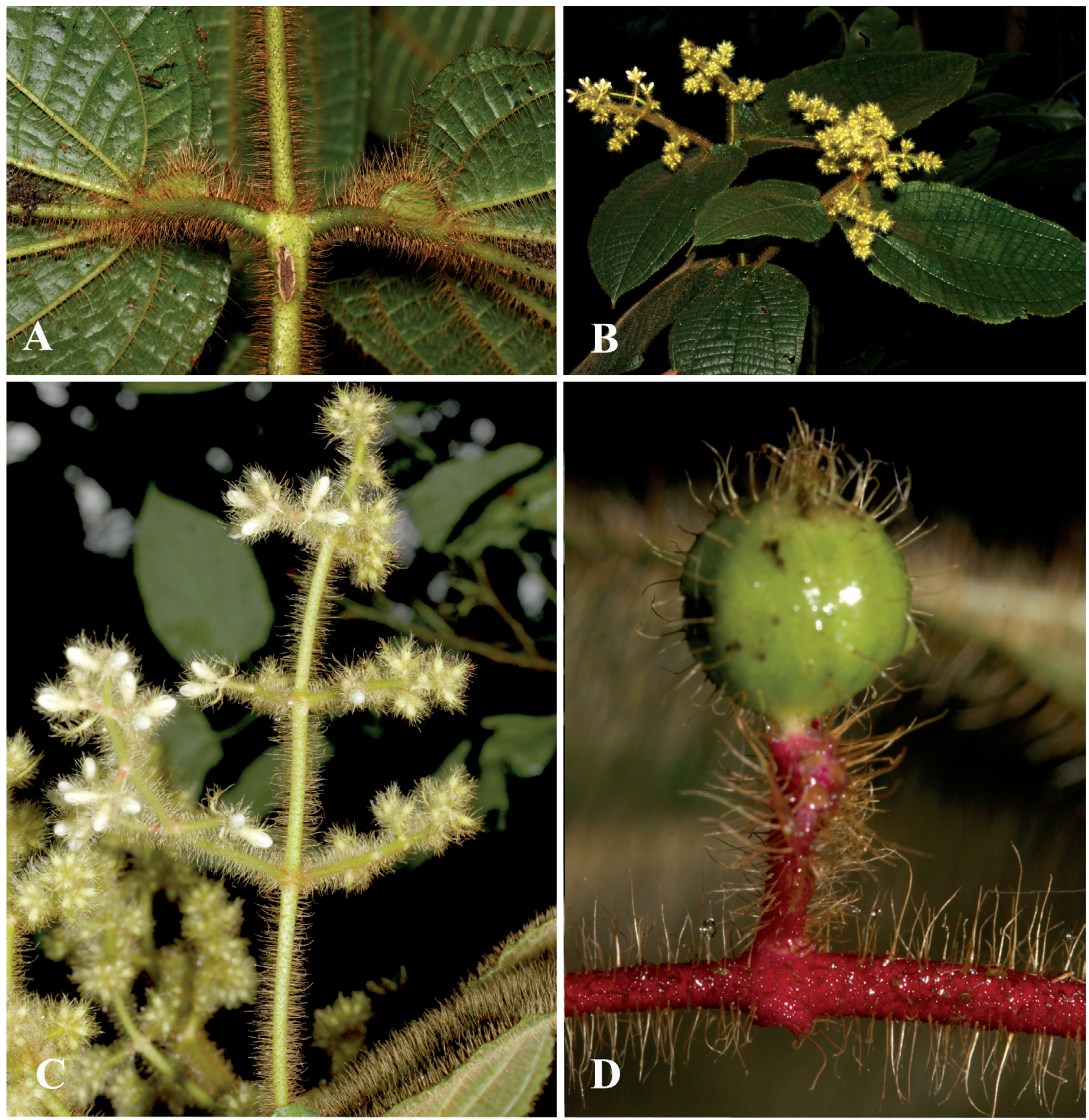

Figura 2. Imágenes de Miconia mailynii. A. Vista de los domacios por el envés foliar. B. Rama florífera. C. Inflorescencia. D. Fruto. Fotos: Julián Aguirre-Santoro de la colección H. Mendoza et al. 19457 (FMB). 

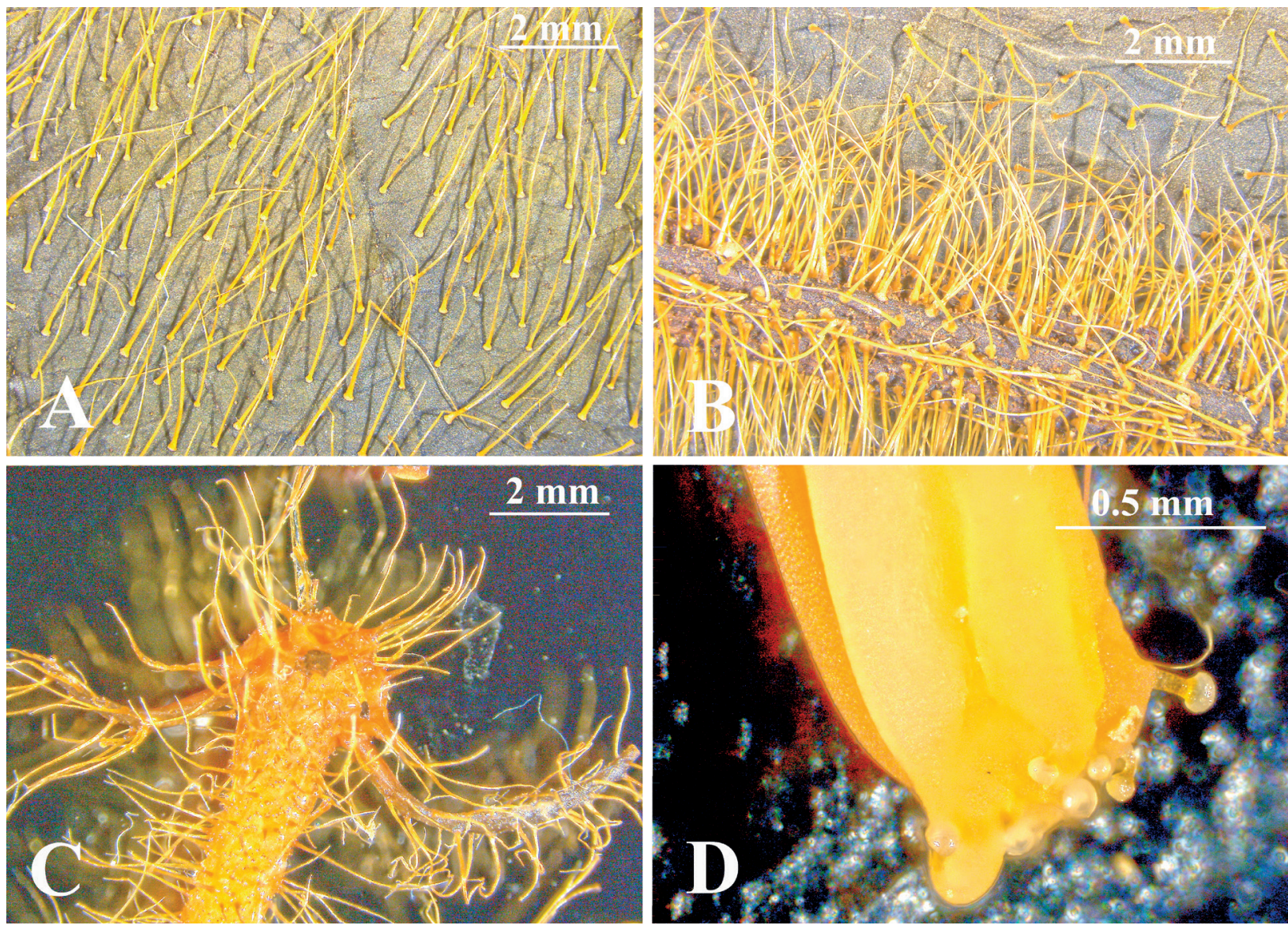

Figura 3. Tricomas en Miconia mailynii. A. Tricomas del haz foliar. B. Tricomas de la vena media por el envés foliar. C). Tricomas del eje de la inflorescencia. D. Tricomas del conectivo basal de la antera. Fotos: a partir de la colección H. Mendoza et al. 19457 (FMB).

\section{Discusión}

Miconia mailynii se reconoce por las hojas levemente anisófilas, domacios en el ápice del pecíolo en ambas hojas del mismo nudo, lámina foliar cordada y basinervada, las inflorescencias terminales paniculadas y el conectivo de la antera basalmente bilobado y con tricomas glandulares. Los taxones más similares morfológicamente corresponden a especies del género Clidemia presentes en Centroamérica. Clidemia taurina de Costa Rica y Panamá, es la especie más cercana, ya que comparte los caracteres de las hojas cordadas con mirmecodomacios en el ápice del pecíolo; no obstante, esta última desarrolla inflorescencia axilares congestiona -das y los pelos de tallos y hojas son reflexos.
Clidemia lanuginosa, de Panamá, es también muy parecida, pero esta última se diferencia principalmente por las hojas isófilas 7-9-plinervias de base redondeada y domacios inmersos en la base de la lámina. Otras especies con alguna semejanza son $C$. ventricosa, de hojas ovadoelípticas con base redondeada e inflorescencias congestionadas y C. spectabilis Gleason (1931: 83), con hojas isofilas e inflorescencias en cabezuelas, axilares o pseudoaxilares.

Clidemia tradicionalmente se ha caracterizado por sus inflorescencias laterales y pétalos de ápice redondeado (Cogniaux, 1891; Judd, 1989); sin embargo, el género es claramente polifilético 


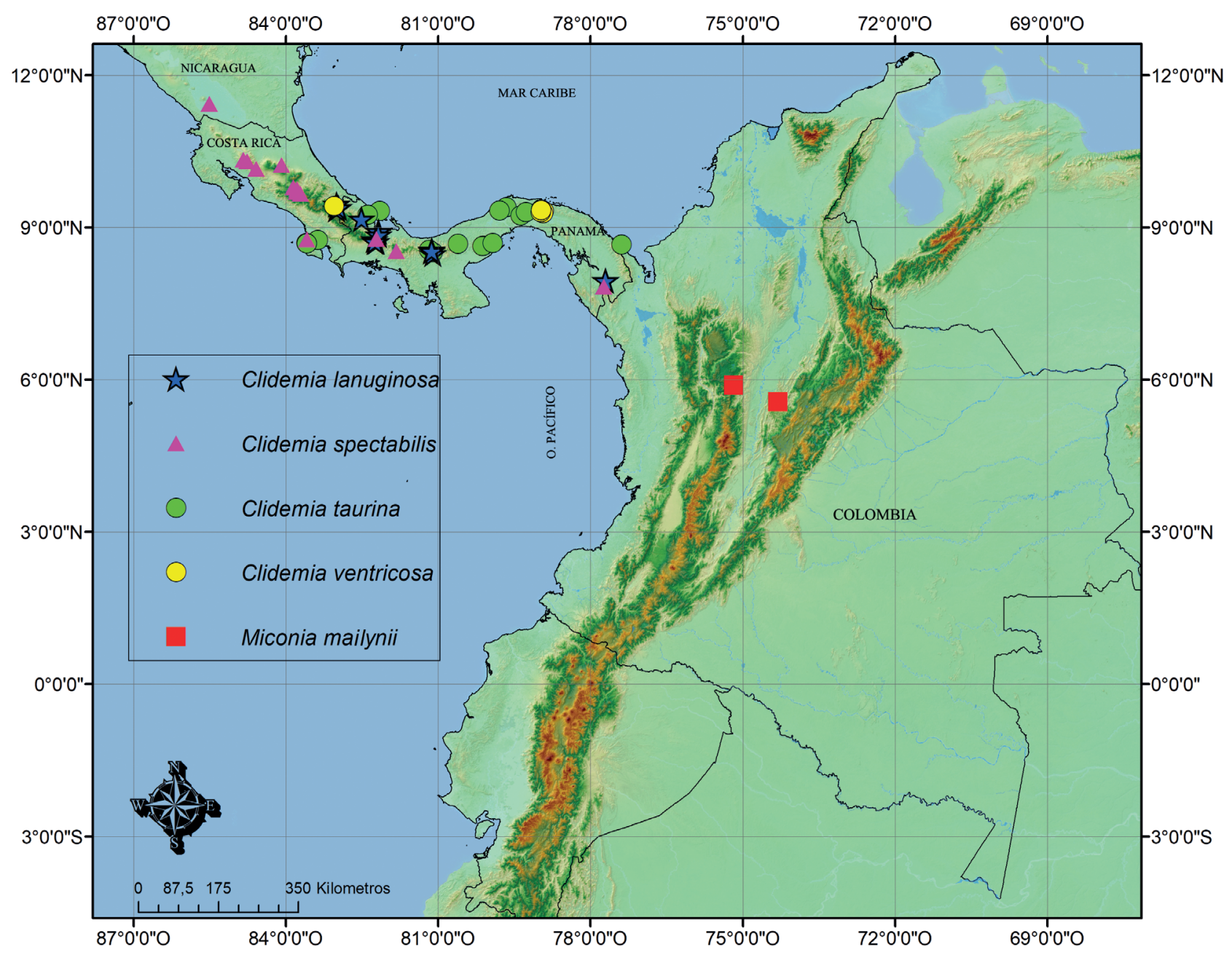

Figura 4. Distribución geográfica de Miconia mailynii y de las especies más similares morfológicamente.

(Michelangeli et al. 2004, 2008; Martin et al. 2008; Goldenberg et al. 2008). La decisión de describir la presente especie bajo el género Miconia obedece a que la mayor parte de las especies de Clidemia probablemente van a ser transferida a Miconia ( $p$. ej. Ionta et al., 2012). Recientes descripciones de especies nuevas asociados a Clidemia se han descrito bajo Miconia (p. ej. Michelangeli y Meier, 2013).

La presencia de mirmecodomacios es uno de los caracteres más llamativos de M. mailynii. Dentro de las Melastomataceae neotropicales se conocen 84 especies asociadas a 11 géneros que desarrollan mirmecodomacios (Michelangeli, 2010). Dentro del género Miconia se conocen 4 especies con este carácter, mientras que en Clidemia 27 especies también lo presentan (Michelangeli, 2010). Miconia mailynii se diferencia de todo este conjunto de especies mirmecofilas por desarrollar los domacios en el ápice del pecíolo, tener las hojas anisófilas de base cordada y poseer inflorescencias terminales.

2. Miconia hernandogarciae Humberto Mend. \& A. Idárraga sp. nov. (Figuras 5, 6 y 7).

Species associated to Octopleura clade; it is recognized by its basinervate leaf, terminal thyrsoid inflorescence, and an ther exhibiting a dorsal connective with a reduced 
tuberculate appendage. Species related to $M$. boekei Gamba y Almeda (2014:67) and M. albertobrenesii Gamba y Almeda (2014:41); however, these two species have plinervate leaf and axillar inflorescence. Also related to M. sessilis Gamba y Almeda (2014:127)but the latter has plinervate leaf with ant domatia.

TIPO: COLOMBIA. Antioquia. Municipio de Carmen de Viboral, vereda El Porvenir, camino a lo largo de filo de la montaña que conduce entre las fincas La Ilusión y La Troja.

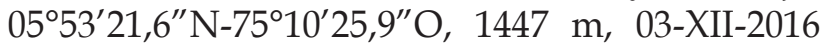
(fl, fr), H. Mendoza et al. 19536 (holotipo: FMB 112361!; isotipos: FMB 112362!, CAUP!, COL!, CUVC!, HUA!).

Arbustos de 2-3 m de altura. Ramas oblongas, levemente aplanadas en los ápices jóvenes; entrenudos 1,7-4,4 cm de largo. Indumento denso rufo en ramas, pecíolo e inflorescencia; tricomas dendríticos amorfos tipo 31 (sensu Wurdack, 1986), 0,1-0,3 mm de largo. Hojas levemente desiguales en tamaño en el mismo nudo. Pecíolo terete hacia la base, acanalado hacia el ápice, en hojas mayores 1,7-3,3 cm de largo, en hojas menores 1-2 cm de largo. Lámina foliar estrechamente elíptica, en hojas mayores 12,5-22 × 3,6-7,2 cm, en hojas menores 8,2-14,5 × 1,8-4,5 cm; base y ápice agudos; margen ligeramente denticulada; haz glabra; envés con indumento denso rufo sobre la nervadura, con tricomas similares a los de las ramas. Venación basal o levemente plinervia a 3 $\mathrm{mm}$ de altura, con un par de venas secundarias acompañando a la vena media. Inflorescencia terminal, en algunas ramas de apariencia axilar por el rápido desarrollo de las yemas vegetativas laterales, paniculada, $6-9 \mathrm{~cm}$ de largo, con 35 a 54 flores; pedúnculo 1,1-1,8 cm de largo; primer internodo 1,9-2,2 cm de largo; eje central con 5-6 nudos de ramificación; paracladios basales 2,6$4,8 \mathrm{~cm}$ de largo; ápices regularmente con una flor; nudo basal con brácteas lineares 5,8-7,6 mm de largo, los nudos superiores con brácteas lineares 2-3,5 $\mathrm{mm}$ de largo, ambas tipos de brácteas con indumento denso rufo en su superficie adaxial; bractéolas triangular lineares, 0,8-1,1 $\mathrm{mm}$ de largo; flores pedicelada y sésiles. Flores 5-mera, diplostémonas, 5,3-5,6 mm de largo sin incluir el pedicelo; pedicelo cuando presente 0,4-0,6 $\mathrm{mm}$ de largo. Hipanto acopado, 1,4-1,6 mm de largo y 2,3-2,5 mm de diámetro; tálamo libre del ovario $0,30-0,33 \mathrm{~mm}$ de largo; externamente 10 costillado y con indumento rufo similar al de las ramas e inflorescencia; internamente con tabiques laminares y ovoides alternos a los pétalos; torus 0,1-0,2 mm de alto. Cáliz truncado, 0,33-0,43 mm de largo; diente dorsal conspicuo, triangular y excediendo levemente la longitud del tálamo, 0,35-0,37 $\mathrm{mm}$ de largo y 0,40-0,44 $\mathrm{mm}$ de ancho, con cubierta rufa similar al hipanto. Corola con pétalos erectos; pétalos 3,6-4,2 × 1-1,4 mm, ovados, con un engrosamiento a manera de quilla en el

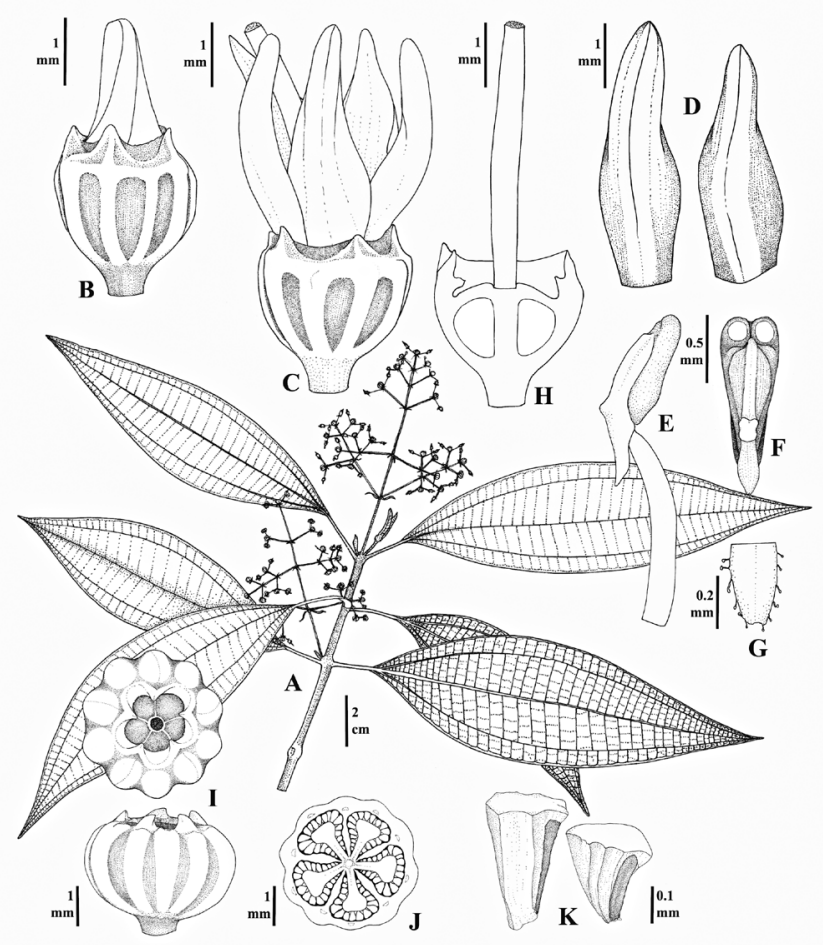

Figura 5. Miconia hernandogarciae. A. Rama con flores y frutos. B. Botón floral. C. Flor. D. Pétalos en vista dorsal. E. Estambre. F. Vista dorsal de la antera. G. Vista dorsal del apéndice conectival basal. H. Corte longitudinal del hipanto-ovario y del estilo. I. Fruto en vista lateral y superior. J. Corte horizontal del fruto. K. Semillas. Basado en H. Mendoza et al. 19536 (FMB). Ilustración: Angélica Ramírez. 
dorso, glabros, blancos. Estambres isomorfos; filamento 1,3-1,5 × 0,2-0,3 mm, laminar, glabro; conectivo con un apéndice basal-dorsal a manera de lengua de $0,25-0,30 \times 0,15-0,18 \mathrm{~mm}$, con tricomas glandulares tipo 3 en la margen de 0,02-0,05 mm de largo; conectivo en la parte dorsal central con un apéndice tuberculado de $0,14 \mathrm{~mm}$ de ancho $\mathrm{y}$ 0,06-0,07 mm de alto; antera obovada, 0,92-0,98 $\mathrm{mm}$ de largo y 0,45-0,47 $\mathrm{mm}$ de ancho, con 2 poros dorsalmente orientados; poros 0,10-0,12 $\mathrm{mm}$ de diámetro. Ovario 5-locular, 1,1-1,2 mm de largo, 3/4 partes fusionado al hipanto; ápice 0,25-0,3 mm de largo, anchamente redondeado, glabro; placentas con rudimentos seminales en todas las superficies. Estilo columnar o levemente ensanchado en la parte central, 4-4,1 mm de largo y 0,35-0,5 mmm de diámetro, glabro; estigma puntiforme, 0,32 $\mathrm{m}$ de diámetro. Fruto 2,2-2,3 mm de largo y 3,7$4 \mathrm{~mm}$ de diámetro, costillado, blanco, con ralos tricomas rufos; con numerosas semillas. Semillas cuneiformes con lados aristados, 0,26-0,37 mmm de largo; testa levemente rugulada, color crema; rafe más oscuro que la testa.

Fenología. Recolectada con flores en diciembre.

Etimología. El epíteto específico es en honor al biólogo Hernando García, subdirector del Instituto de Investigación de Recursos Biológicos

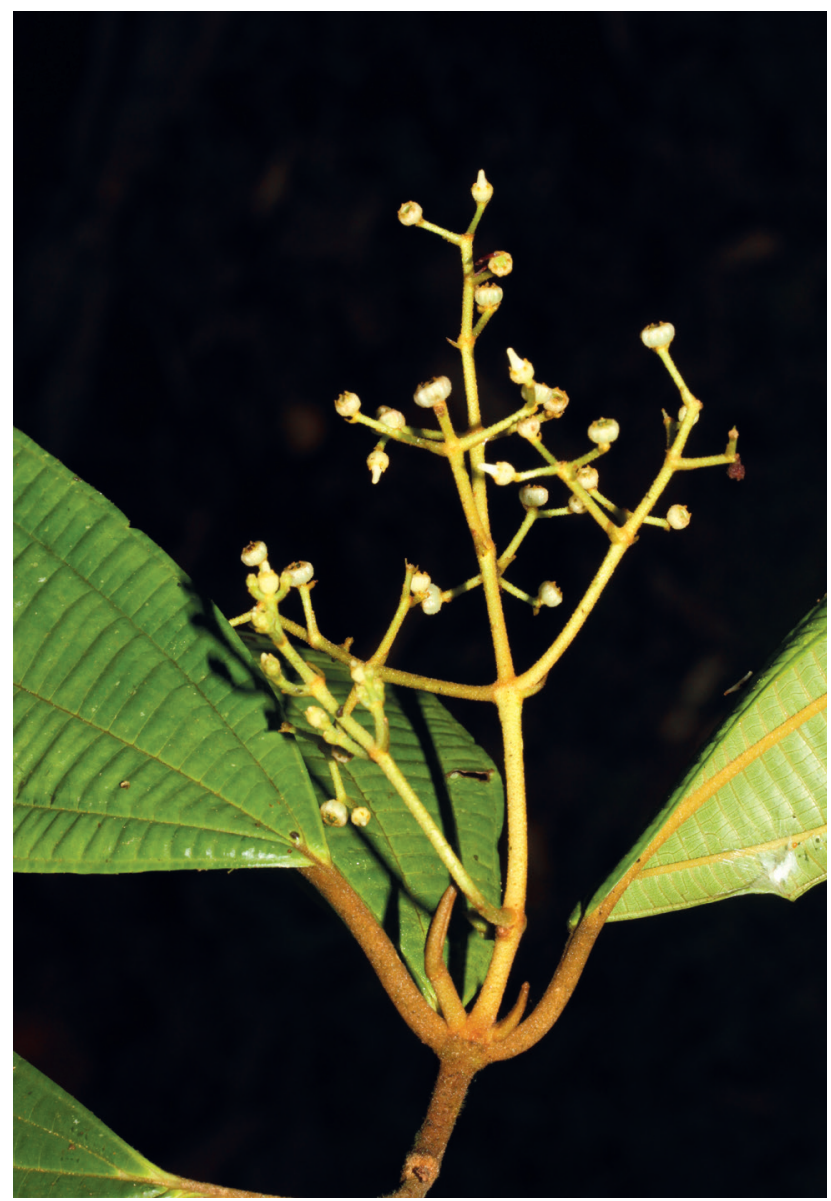

Figura 6. Imagen de Miconia hernandogarciae. Foto: Julián Aguirre Santoro de la colección H. Mendoza et al. 19536 (FMB).
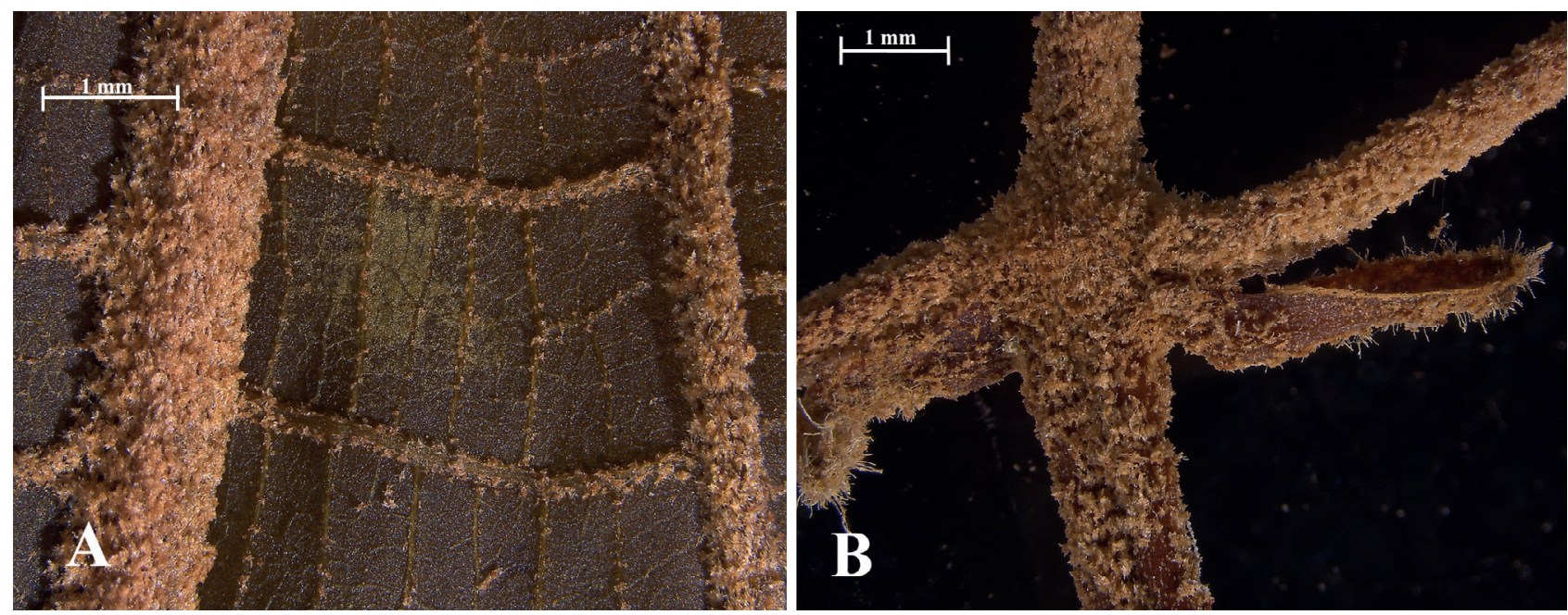

Figura 7. Tricomas en Miconia hernandogarciae. A. Tricomas del envés foliar. B. Tricomas del eje de la inflorescencia. Fotos: colección H. Mendoza et al. 19536 (FMB). 
Alexander von Humboldt, amante de las plantas y gestor de la expedición Colombia BIOAntioquia.

Distribución y hábitat. Posiblemente se encuentra en la franja de bosque subandino en los flancos de las cordilleras Central y Oriental que colinda con el Magdalena Medio en Antioquia y Santander. No obstante, hasta el momento solo se conoce de la localidad tipo (Figura 8). Crece en zonas de bosque en buen estado de conservación.

Estado de conservación. Miconia hernandogarciae se conoce solo de la localidad tipo en el piedemonte que colinda con el Magdalena Medio. Estas especie crece en una franja altitudinal estrecha, está asociada a zonas de bosque y es poco frecuente en el área de su procedencia. Cumple el criterio B1biii de la IUCN $(2001,2010)$, por lo que se recomienda catalogarla como una especie En peligro (EN).

\section{Discusión}

Miconia hernandogarciae se asocia al clado Octopleura recientemente revisado por Gamba y Almeda (2014). Este clado es un grupo natural que comprende 33 especies neotropicales, siendo la mayoría de sus taxones descritos originalmente bajos los géneros Ossaea y Clidemia pero actualmente transferidos a Miconia (Gamba y Almeda, 2014).

Este clado se caracteriza por los estambres isomorfos, anteras con uno o dos poros dorsal-

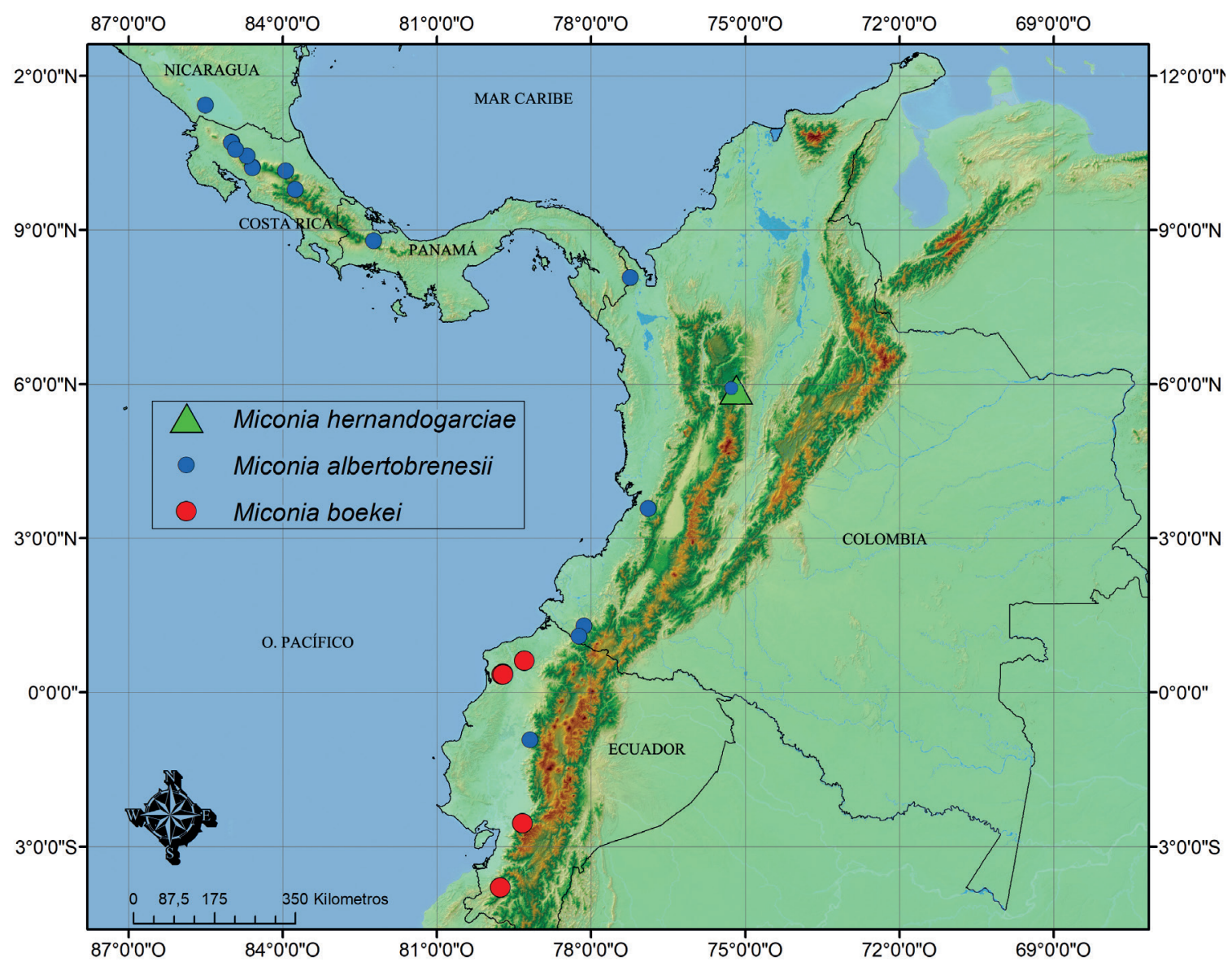

Figura 8. Distribución geográfica de Miconia hernandogarciae y de las especies más similares morfológicamente. 
mente orientados, conectivo prolongado dorsobasalmente y con glándulas, e hipanto costillado (Gamba y Almeda, 2014). Se distribuye desde el suroriente de México hasta el centro-norte de Suramérica (Colombia, Ecuador, Perú, Venezuela y Brasil), con una especie de amplia distribución que alcanza a las Antillas (Gamba y Almeda, 2014). Colombia, con más de 25 especies, es el país con mayor diversidad de este grupo, con especies principalmente distribuidas en la región Pacífica y los Andes.

Dentro del clado Octopleura, Miconia hernandogarciae se asocia al subclado Variabilis $\mathrm{y}$ se asemeja a $M$. boekei de Ecuador y $\mathrm{M}$. albertobrenesii que crece desde Nicaragua hasta Ecuador. Se separa de las dos anteriores especies por las inflorescencias terminales y las hojas basalmente nervadas. Otra característica que distingue a esta nueva especie es una protuberancia en la parte central del conectivo dorsal de la antera.

\section{Agradecimientos}

Expresamos sinceros agradecimientos al Instituto de Investigación de Recursos Biológicos Alexander von Humboldt y especialmente a los coordinadores del proyecto Colombia BIO: Hernando García, Carlos Lasso, Mailyn González y Javier Barriga. Extendemos nuestros agradecimientos a la comunidad de la vereda El Porvenir en Antioquia quien nos apoyó durante la expedición de Colombia $\mathrm{BIO}$, a las auxiliares del herbario FMB Aura Robles y Janeth Robles por el procesamiento de las colecciones y a Andrés Rymel Acosta por la elaboración de los mapas de distribución. A Colciencias, financiadora del Convenio Especial ColombiaBIO COLCIENCIAS-IAVH FP44842-109-2016 (IAVH 16-062).

\section{Referencias}

Almeda, F. (2004). Novelties and nomenclatural adjustments in the neotropical genus Clidemia (Melastomataceae: Miconieae). Proceedings of the California Academy of Sciences, ser. 4, 55 (4): 89124.
Almeda, F. (2009). Melastomataceae. En Davidse, G., Sousa-Sânchez, M., Knapp, S. y Chiang, F. (Eds.). Flora Mesoamericana: Cucurbitaceae a Polemoniaceae, 4 (1): 164-338.

Almeda, F., Alvear, M., Mendoza-Cifuentes, H., Penneys, D. S. y Michelangeli, F. (2016). Melastomataceae. Pp. 1585-1664. En Bernal, R., Gradstein, S. R. y Celis, M. (Eds.). Catálogo de plantas y líquenes de Colombia. Bogotá, Colombia: Instituto de Ciencias Naturales, Universidad Nacional de Colombia.

Cogniaux, C. A. (1891). Melastomataceae. En Candolle, A. L. P. P. de y A.C. P. Candolle de (Eds.) Monographiae Phanerogamarum 7. G. Masson, Paris. 1256 pp.

Gleason, H. A. (1931). The relationships of certain myrmecophilous Melastomes. Bulletin of the Torrey Botanical Club, 58 (2): 83-84.

Gleason, H. A. (1950). Observations on tropical American Melastomes. Phytologia, 3 (7): 360.

Gamba, D. y Almeda, F. (2014). Systematics of the Octopleura Clade of Miconia (Melastomataceae: Miconieae) in Tropical America. Phytotaxa, 179 (1): 1-174.

Goldenberg, R., Penneys, D. S., Almeda, F., Judd, W. S. y Michelangeli, F. A. (2008). Phylogeny of Miconia (Melastomataceae): Patterns of stamen diversification in a megadiverse neotropical genus. International Journal of Plant Sciences, 169: 963-979.

Ionta, G., Judd, W. S., Skean, J. D. y McNullen, C. K. (2012). Two new species of Miconia sect. Sagraea (Melastomataceae) from the Macaya Biosphere Reserve, Haiti, and twelve relevant new species combinations. Brittonia, 64: 61-72.

IUCN. (2001). IUCN Red List Categories and Criteria: Version 3.1. IUCN Species Survival Commission. IUCN, Gland, Switzerland and Cambridge, UK.

IUCN Standards and Petitions Working Group. (2010). Guidelines for Using the IUCN Red List Categories and Criteria. Version 8.0. Prepared by the Standards and Petitions Subcommitee in March 2010. 
Judd, W. S. (1989). Taxonomic studies in the Miconieae (Melastomataceae) III. Cladistic analysis of axillary-flowered taxa. Annals of the Missouri Botanical Garden, 76: 476-495.

Martin, C.V., Little, D. P., Goldenberg, R. y Michelangeli, F. A. (2008). A phylogenetic evaluation of Leandra (Miconieae, Melastomataceae): a polyphyletic genus where the seeds tell the story, not the petals. Cladistics, 24: 315-327.

Michelangeli, F.A., Penneys, D. S., Giza, J., Soltis, D., Hils, M. H. y Skean, J. D. (2004). A preliminary phylogeny of the tribe Miconieae (Melastomataceae) based on nrITS sequence data and its implications on inflorescence position. Taxon, 53: 279-290.

Michelangeli, F.A., Judd, W. S., Penneys, D. S., Skean, J. D., Bécquer-Granados, E. R., Goldenberg, R. y Martin, C. V. (2008). Multiple events of dispersal and radiation of the tribe Miconieae (Melastomataceae) in the Caribbean. Botanical Review, 74: 53-77.

Michelangeli, F. A. (2010). An annotated list and key of neotropical myrmecophilous Melastomataceae. Proceedings of the California Academy of Sciences, 61: 407-447.

Michelangeli, F. A. y Meier, W. (2013). A new anisophyllous species of Miconia (Melastomataceae: Miconieae) from the Coastal

Humberto Mendoza-Cifuentes

Herbario Federico Medem,

Instituto de Investigación de Recursos Biológicos

Alexander von Humboldt

Villa de Leyva, Boyacá, Colombia

hmendoza@humboldt.org.co

Julián Aguirre-Santoro

Instituto de Ciencias Naturales,

Universidad Nacional de Colombia

Bogotá, Colombia

jaaguirresa@gmail.com

Álvaro Idárraga

Herbario HUA, Universidad de Antioquia

alvaro.idarraga@gmail.com
Cordillera in northern Venezuela. Phytotaxa, 79 (1): 37-44.

Michelangeli, F. A., Nichole, T. y Kimberly, W. (2013). The PBI Miconieae project website: towards complete web-based monograph of the tribe Miconieae (Melastomataceae). Resumen de presentación oral. Recuperado de: http://2013. botanyconference.org/engine/search/index. php? func $=$ detail $\&$ aid $=689$

Michelangeli, F. A., Almeda, F., Alvear, M., Bécquer, E. R., Burke, J., Caddah, M. K., Goldenberg, R., Ionta, G. M., Judd, W. S., Majure, L. C., Meirelles, J., Nicolas, A. N., Ocampo, G., Penneys, D. S., Dan Skean Jr., J. y Ulloa, C. (2016). Proposal to conserve Miconia, nom. cons. against the additional names Maieta and Tococa (Melastomataceae: Miconieae). Taxon, 65 (4): 892893.

Van der Hammen, T. y Rangel-Ch, J. O. (1997). El estudio de la vegetación en Colombia. Pp. 17-57. En Rangel, O., Lowy, P. D. y Aguilar, M. (Eds.). Colombia - Diversidad Biótica II - Tipos de vegetación. Bogotá, Colombia: Instituto de Ciencias Naturales/Universidad Nacional de Colombia; Instituto de Hidrología, Meteorología y de Estudio Ambientales IDEAM.

Wurdack, J. J. (1986). Atlas of hairs for Neotropical Melastomataceae. Smithsonian Contributions to Botany, 63: 1-80.
Dos nuevas especies de Miconia

(Melastomataceae) del piedemonte oriental de la cordillera Central de Antioquia, Colombia

Citación del artículo: Mendoza-Cifuentes, H., Aguirre-Santoro, J. y Idárraga, A. (2018). Dos nuevas especies de Miconia (Melastomataceae) del piedemonte oriental de la cordillera Central de Antioquia, Colombia. Biota Colombiana, 19 (Sup. 1): 17-28. DOI: 10.21068/c2018.v19s1a03.

Recibido: 11 de julio de 2017

Aprobado: 30 de septiembre de 2017 


\section{Guía para autores \\ (http://revistas.humboldt.org.co/index.php/biota)}

\section{Envío del manuscrito}

El envío de un manuscrito implica la declaración explícita por parte del autor o los autores de que este no ha sido previamente publicado, ni aceptado para su publicación en otra revista u otro órgano de difusión científica. Todas las contribuciones son de la entera responsabilidad de sus autores y no del Instituto de Investigación de Recursos Biológicos Alexander von Humboldt, ni de la revista o sus editores.

Los trabajos pueden estar escritos en español, inglés o portugués, y no deben exceder las 40 páginas (párrafo espaciado a 1,5 líneas), incluyendo tablas, figuras y anexos. De particular interés para la revista son las descripciones de especies nuevas para la ciencia, nuevos registros geográficos, listados de especies temáticos o regionales, inventarios, bases de datos relacionados con biodiversidad, colecciones biológicas y reportes de muestreo.

Se reciben manuscritos que sean artículos científicos de investigación, así como notas de actualidad, reseñas, novedades bibliográficas y artículos de datos.

Los trabajos deben ser entregados a través del portal en línea (http://revistas.humboldt.org.co/index. php/biota) de la revista, siguiendo los pasos de registro como usuario. Todo el proceso editorial se desarrolla a través de esa plataforma.

\section{Evaluación del manuscrito}

Los manuscritos sometidos serán revisados por mínimo dos pares científicos calificados, cuya respuesta final de evaluación puede ser: a) publicado sin ningún cambio (se asume que no existe ningún cambio, omisión o adición al artículo, y que se recomienda su publicación en la forma actualmente presentada); b) aceptación condicional (se acepta y recomienda el artículo para su publicación solo si se realizan los cambios indicados por el evaluador, sean correcciones menores y no es necesaria una segunda re- visión o correcciones mayores y sí es necesaria una segunda revisión); y c) rechazo (cuando el evaluador considera que los contenidos o forma de presentación del artículo no se ajustan a los requerimientos y estándares de calidad de Biota Colombiana). Para proseguir con el proceso editorial para publicación, el manuscrito debe haber sido aceptado por el mínimo de dos pares científicos.

\section{Preparación del manuscrito}

Para la elaboración de los textos del manuscrito se debe usar un procesador de palabras (preferiblemente Word); los listados (a manera de tabla) deben ser elaborados en una hoja de cálculo (preferiblemente Excel). Para someter un manuscrito es necesario además anexar una carta de intención en la que se indique claramente:

1. Nombre completo del (los) autor (es), afiliaciones institucionales y direcciones para envío de correspondencia (es indispensable suministrar una dirección de correo electrónico para comunicación directa).

2. Título completo del manuscrito.

3. Nombres, tamaños y tipos de archivos suministrados.

4. Explicación concisa y clara, que no sobrepase tres líneas, explicando por qué el manuscrito en cuestión es ideal para que sea publicado en una revista como Biota Colombiana.

5. Lista mínimo de cuatro revisores sugeridos que puedan evaluar el manuscrito, con sus respectivas direcciones electrónicas.

**Para información sobre la preparación de un artículo de datos, continúe a la sección "Particularidades de los artículos de datos $\rightarrow$ Preparación de un artículo de datos" dentro de esta Guía de autores. 


\section{Lenguaje del manuscrito}

- Los manuscritos enviados a Biota Colombiana deben tener como requerimiento mínimo el uso adecuado del lenguaje en el que estén escritos, sea español, portugués o inglés, para asegurar la comunicación efectiva del artículo a los lectores.

- El estilo utilizado en el lenguaje del manuscrito enviado debe seguir las formalidades comúnmente aceptadas en escritos científicos y siempre buscar la claridad, concisión y cohesión en su expresión.

- Se sugiere utilizar guías de buena ortografía, redacción y estilo para el idioma en el que se escoja escribir.

\section{Especificaciones de formato}

- Para la presentación del manuscrito configure las páginas de la siguiente manera: hoja tamaño carta, márgenes de $2,5 \mathrm{~cm}$ en todos los lados, interlineado 1,5 y alineación hacia la izquierda (incluyendo título y bibliografía).

- Todas las páginas de texto deben numerarse en la parte inferior derecha de la hoja.

- Use letra Times New Roman o Arial, tamaño 12 puntos en todos los textos. Máximo 40 páginas, incluyendo tablas, figuras y anexos. Para tablas cambie el tamaño de la fuente a 10 puntos. Evite el uso de negritas o subrayados.

- Escriba los nombres científicos de géneros, especies y subespecies en cursiva (itálica). Proceda de la misma forma con los términos en latín (por ej. sensu, et al.). No subraye ninguna otra palabra o título. No utilice notas al pie de página.

- En cuanto a las abreviaturas y sistema métrico decimal, utilice las normas del Sistema Internacional de Unidades (SI) recordando que siempre se debe dejar un espacio libre entre el valor numérico y la unidad de medida (por ej. 16 $\mathrm{km}, 23{ }^{\circ} \mathrm{C}$ ). Para medidas relativas como $\mathrm{m} / \mathrm{seg}$., use m.seg-1.

- Escriba los números del uno al diez siempre con letras, excepto cuando preceden a una unidad de medida (por ej. $9 \mathrm{~cm}$ ) o si se utilizan como marcadores (por ej. parcela 2, muestra 7). Los números mayores a diez deben ser escritos con los símbolos numéricos arábigos. Si en el mismo párrafo se utilizan cifras menores a diez y cifras mayores a diez, se deben unificar para dejar las cifras solo con símbolos numéricos arábigos.

- No utilice punto para separar los millares, millones, etc (por ej. 54000). Utilice la coma para separar en la cifra la parte entera de la decimal (por ej. 3,1416) cuando el texto es en español. En el caso del inglés, los decimales se separan con puntos (por ej. 3.1416). Enumere las horas del día de 0:00 a 24:00.

- Exprese los años con todas las cifras sin demarcadores de miles (por ej. 1996-1998). En español los nombres de los meses y días (enero, julio, sábado, lunes) siempre se escriben con la primera letra minúscula, en inglés se escriben con la primera letra mayúscula (January, July, Saturday, Monday).

- Los puntos cardinales (norte, sur, este y oeste) en español siempre deben ser escritos en minúscula, a excepción de sus abreviaturas N, S, E, O (en inglés $W$ ), y cuando son referidos como puntos o hacen parte de un nombre propio (p.e. cordillera Oriental). La indicación correcta de coordenadas geográficas es como sigue: $02^{\circ} 37^{\prime} 53^{\prime \prime} \mathrm{N}-$ $56^{\circ} 28^{\prime} 53^{\prime \prime} \mathrm{O}$. La altitud geográfica se citará como se expresa a continuación: 1180 m s.n.m. y en inglés $1180 \mathrm{~m}$ a.s.l.

- Las abreviaturas se deben explicar la primera vez que son usadas.

- Al citar las referencias en el texto, siga las normas APA (Manual de Publicaciones de la American Psychological Association, Sexta Edición). Incluya los apellidos de los autores en caso de que sean uno o dos, y el apellido del primero seguido por et al. (en cursiva) cuando sean tres o más. En el caso de dos autores, los apellidos deben ser separados por la palabra "y" (por ej. Cochran y Goin, 1970). En inglés, los apellidos deben estar separados por " $\&$ ". Si menciona varias referencias, éstas deben ser ordenadas cronológicamente y separadas por punto y coma (por ej. Rojas, 1978; Bailey et al., 1983; Sephton, 2001, 2001). Inserte una coma después de los nombres de los autores y antes del año de la referencia (Acevedo, 2009). 
- Las referencias que son autoridad taxonómica de descripción no deben ser incluidas en la lista de referencias al final pero sí en el texto.

- Refiera las figuras (gráficas, diagramas, ilustraciones y fotografías) sin abreviación (por ej. Figura 3) al igual que las tablas (por ej. Tabla 1). Gráficos y figuras deben presentarse con tipo y tamaño de letra uniforme.

- Las figuras deben ser nítidas y de buena calidad, evitando complejidades innecesarias (por ej. tridimensionalidad en gráficos de barras, marcos o efectos tridimensionales); si es posible use solo colores sólidos en lugar de tramas. Las letras, números o símbolos de las figuras deben ser de un tamaño adecuado de manera que sean claramente legibles una vez reducidas.

- Cada figura debe estar insertada en el texto, y adicionalmente se debe enviar su archivo aparte en alta calidad en el paso de "Cargar los archivos complementarios". Para el caso de las fotografías y figuras digitales es necesario que estas sean guardadas como formato tiff, jpg o png con una resolución de 300 dpi.

- Las tablas y anexos deben ser simples en su estructura (marcos) y estar unificados. Haga las llamadas a pie de página de tabla con letras ubicadas como superíndice. Evite tablas grandes sobrecargadas de información y líneas divisorias o presentadas en forma compleja.

\section{Secciones del manuscrito}

** Para información sobre las secciones de un artículo de datos, continúe a la sección "Particularidades de los artículos de datos $\rightarrow$ Secciones de un artículo de datos" dentro de esta Guía de autores.

- Los manuscritos deben llevar el siguiente orden: título, resumen y palabras clave, abstract y keywords, introducción, materiales y métodos, resultados, discusión, conclusiones (optativo), agradecimientos (optativo) y referencias. Seguidamente, presente una página con la lista de tablas, figuras y anexos.

- Las secciones del manuscrito en el texto deben ir en negrilla, con solo la primera letra en mayúscula. Si necesita agregar subtítulos a las secciones, estos deben ir en la misma línea en la que comienza el texto del párrafo, separados por un punto del resto del texto, y en negrilla.

Título: conciso y explicativo, debe informar sobre el contenido del manuscrito.

Resumen: da un resumen de máximo 200 palabras sobre el manuscrito, en el que se debe incluir el objetivo, métodos, resultados y conclusiones principales del manuscrito. Si se presenta algo que sea novedoso o excepcional, se debe hacer mención aquí. El resumen se debe escribir en dos idiomas (español o portugués y abstract en inglés).

Palabras clave: máximo cinco palabras clave, complementarias al título del artículo, en español o portugués e inglés, separadas por un punto entre cada término. Deben presentarse en orden alfabético. Se sugiere el uso de tesauros temáticos para encontrar sinónimos y términos adecuados.

Introducción: presenta el tema y da el contexto necesario para el desarrollo del manuscrito. El propósito u objetivo principal del trabajo debe hacerse explícito en esta sección.

Materiales y métodos: hace una descripción detallada del procedimiento, incluyendo los materiales, lugar, fechas, métodos estadísticos, etc. que se utilizaron en el trabajo. Debe ser lo suficientemente completo para que otros investigadores puedan replicar el trabajo y si se usa una metodología novedosa debe explicarse y sustentarse.

Resultados: presenta los hallazgos del trabajo de manera organizada y con uso adecuado de figuras. Evitar la inclusión de tablas muy extensas en esta sección y más bien incluir como anexos si es el caso.

Discusión: se destacan los puntos más relevantes, polémicos o novedosos del trabajo y se explican los resultados principales en relación a la importancia o aportes del trabajo en su área.

Conclusiones: reflexiones finales sobre el trabajo con relación a su propósito y objetivos, frecuentemente direccionando hacia acciones e investigaciones futuras.

Agradecimientos: Párrafo sencillo y conciso entre el texto y la lista de referencias. Mencione fuentes 
de financiación o apoyo que recibió el proyecto. Evite títulos como Dr., Lic., TSU, etc.

Referencias: La revista sigue las normas de citación APA (Manual de Publicaciones de la American Psychological Association, Sexta Edición). La lista de las referencias contiene únicamente aquellas citadas en el texto. Ordénelas alfabéticamente por autores y cronológicamente para un mismo autor. Si hay varias referencias de un mismo autor(es) en el mismo año, añada las letras a, b, c, etc. al año. No abrevie los nombres de las revistas. Incluya todos los autores de la referencia. Presente las referencias al final del manuscrito.

\section{Ejemplos de citación}

\section{Artículo en revistas:}

Antonelli, A., Nylander, J. A., Persson, C. y Sanmartín, I. (2009). Tracing the impact of the Andean uplift on Neotropical plant evolution. Proceedings of the National Academy of Sciences, 106(24): 9749-9754.

\section{Libros:}

Gutiérrez, F. P. (2010). Los recursos hidrobiológicos $y$ pesqueros en Colombia. Bogotá: Instituto de Investigación de Recursos Biológicos Alexander von Humboldt. 118 pp.

\section{Tesis:}

Cipamocha, C. A. (2002). Caracterización de especies y evaluación trófica de la subienda de peces en el raudal Chorro de Córdoba, bajo río Caquetá, Amazonas, Colombia. (Trabajo de grado). Bogotá D. C.: Universidad Nacional de Colombia, Facultad de Ciencias, Departamento de Biología. 160 pp.

\section{Informes técnicos:}

Andrade, G. I. (2010). Gestión del conocimiento para la gestión de la biodiversidad: bases conceptuales y propuesta programática para la reingeniería del Instituto Humboldt. (Informe técnico). Bogotá D. C.: Instituto de Investigación de Recursos Biológicos Alexander von Humboldt. 80 pp.

\section{Capítulo en libro o en informe:}

Fernández F., Palacio, E. E. y MacKay, W. P. (1996). Introducción al estudio de las hormigas
(Hymenoptera: Formicidae) de Colombia. En Amat, G. D., Andrade, G. y Fernández, F. (Eds.). Insectos de Colombia. Estudios Escogidos. Pp: 349-412. Bogotá: Academia Colombiana de Ciencias Exactas, Físicas y Naturales y Centro Editorial Javeriano.

\section{Resumen en congreso, simposio, talleres:}

Señaris, J. C. (2001). Distribución geográfica y utilización del hábitat de las ranas de cristal (Anura; Centrolenidae) en Venezuela. Trabajo presentado en Programa y Libro de Resúmenes del IV Congreso Venezolano de Ecología, Mérida, Venezuela. p. 124.

\section{Ley o Decreto:}

Congreso de Colombia. (8 de febrero de 1994) Ley General de Educación. [Ley 115 de 1994]. DO: 41.214.

\section{Páginas web:}

No serán incluidas en la lista de referencias, sino que se señalan claramente en el texto al momento de mencionarlas.

\section{Particularidades de los artículos de datos}

Un Artículo de Datos o Data Paper es un tipo de publicación académica que surgió como un mecanismo para incentivar la publicación de datos sobre biodiversidad. Es un medio para generar reconocimiento académico y profesional adecuado a todas las personas que intervienen, de una manera u otra, en la gestión de información sobre biodiversidad, y además sirve para destacar la existencia y relevancia de los conjuntos de datos frente el resto de la comunidad científica.

Como su nombre lo sugiere, este tipo de artículos se basan en la descripción de un conjunto de datos primarios, y aunque no es una investigación científica sensu stricto, se espera que contengan información acerca de la historia del conjunto de datos (propósito del mismo, metodología sobre la toma de los datos, financiadores, coberturas taxonómicas y geográficas, etc.) y sobre su valor y utilidad (básica o aplicada) para la comunidad científica (Chavan y Penev, 2011) ${ }^{1}$. Lo novedoso y ventajoso de este mo-

\footnotetext{
${ }^{1}$ Chavan, V. y Penev, L. (2011). The data paper: The mechanism to incentivize data publishing in biodiversity science. BMC Bioinformatics 2011, 12(Sup. 15): S2
} 
delo de publicación es que el manuscrito siempre está vinculado al conjunto de datos, a través de un enlace a un repositorio web persistente y confiable, el IPT (Integrated Publishing Toolkit). Adicionalmente los metadatos que describen ese conjunto de datos y que están documentados en la misma herramienta, deben citar el artículo de datos.

Se recomienda someter un artículo de datos, cuando los datos a los que hace referencia son primarios, originales y están restringidos temporal y metodológicamente, se encuentran disponibles en agregadores de datos como el SiB Colombia y GBIF, y pueden ser estructurados con el estándar Darwin Core (DwC) como en el caso de:

- Observaciones de un proyecto particular

- Colecciones biológicas

- Listados de especies

- Datos genómicos

- Eventos de muestreo

- Inventarios

- Bases de datos

- Rasgos funcionales

Los conjuntos de datos que no cumplan estas características, no serán aceptados para publicación como artículo de datos. Tal es el caso de compilaciones de registros biológicos de fuentes secundarias (por ej., literatura).

\section{Preparación de un artículo de datos (publicación de datos y creación del manuscrito)}

Un artículo de datos busca describir todos los tipos de recursos de datos sobre biodiversidad. De esta manera, el artículo de datos siempre está enlazado al conjunto de datos que describe a través de una URL o DOI.

A continuación encontrará el procedimiento de cómo generar y someter un manuscrito para artículo de datos usando las herramientas y modelo de publicación del SiB Colombia. Cabe resaltar que la revista también recibe artículos de datos que tengan conjuntos de datos publicados en otras plataformas reconocidas, siempre y cuando cuenten con los requisitos de ser repositorios confiables y tengan un enlace IPT disponible. Igualmente, las secciones de los manuscritos enviados deben seguir las enunciadas en la Tabla 1.

Los artículos de datos, como los demás tipos de manuscritos enviados a la revista, serán sometidos a evaluación por pares y deben cumplir las mismas especificaciones de formato, normas de citación y uso del lenguaje, además de incluir una carta de intención, como se menciona en esta Guía de autores. Adicionalmente, durante la evaluación del manuscrito, los datos descritos deben estar disponibles en línea de manera abierta en un repositorio público y con una licencia robusta de atribución y uso.

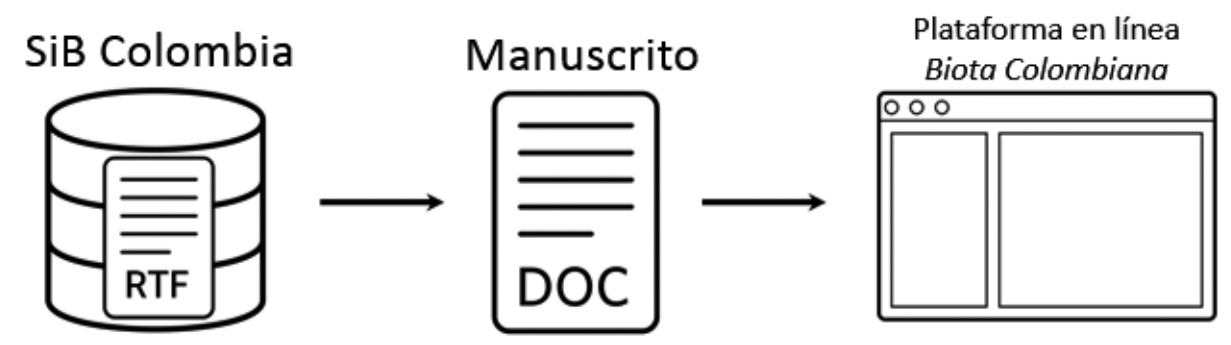

Figura 1. Proceso general de sometimiento de un artículo de datos desde el SiB Colombia a la revista Biota Colombiana. 
Paso 1.

Publicación de los datos a través del SiB Colombia

El SiB Colombia cuenta con un modelo de publicación de datos que hace uso del IPT como herramienta. Desde el IPT, es posible generar una primera versión del manuscrito a partir de los metadatos en formato de texto enriquecido (RTF), siempre $y$ cuando el conjunto de datos respectivo ya haya sido indexado por el SiB Colombia y cuente con los metadatos suficientes (el proceso de publicación de datos a través del SiB Colombia puede ser consultado en https://www.sibcolombia.net/).

A. Registro de organización. Para poder publicar a través del SiB Colombia, es necesario que la organización esté registrada como socio publicador. Se puede comprobar en este enlace si ya es así. En caso contrario, es muy sencillo hacer parte de la red de socios, solo se debe diligenciar el Formulario de Registro.

B. Estandarización de datos. Los datos se deben estructurar en una tabla plana haciendo uso del estándar Darwin Core (DwC). Para esto, se puede descargar la plantilla que se adapte a el tipo de datos o generar una plantilla a través del Generador de Plantillas Excel.

C. Calidad de sus datos. Se debe verificar y mejorar la calidad de los datos haciendo uso de herramientas para identificar, limpiar y corregir posibles errores geográficos, taxonómicos o de formato, entre otros.

D. Cargar datos en línea. El IPT es una herramienta que facilita compartir diferentes tipos de datos relacionados con la biodiversidad siempre y cuando estos se encuentren estructurados según el estándar DwC. Para cargar datos en el IPT, debe contar con una cuenta de usuario en uno de los IPT disponibles por el SiB Colombia. Si aún no se tiene una cuenta, puede contactar al Equipo Coordinador del SiB Colombia (EC-SiB) y solicitarla a través del correo electrónico sib@humboldt.org.co.

E. Mapear datos. Una vez se haya cargado el conjunto de datos, se debe verificar que estén siendo leídos en correspondencia con los elementos DwC. El manual de usuario del IPT está disponible para más información o se puede contactar al EC-SiB.
F. Creación de metadatos. La estructura de los metadatos se parece en gran medida a un artículo de investigación tradicional. Estos metadatos mantienen la estructura general de un artículo de datos y facilitan la generación del mismo. En la sección Metadatos del IPT, se debe documentar toda la información que permite dar un contexto a los datos. En total hay 12 secciones para ingresar información que describe el conjunto de datos. El manual de usuario del IPT está disponible para más información o se puede contactar al EC-SiB.

G. Publicar el recurso y notificar al EC-SiB. Una vez completados los pasos anteriores, el IPT activará la opción 'Publicar'. Se debe hacer clic en este botón y enviar un correo a sib@humboldt.org.co para notificar al EC-SiB de su publicación. El correo debe tener como asunto "Recurso publicado" y contener:

- Nombre

- Nombre de organización

- Nombre del recurso publicado

- URL de la vista general del recurso después de publicado

En este punto los datos serán indexados por el SiB Colombia y GBIF, y contarán con un identificador persistente DOI.

\section{Paso 2}

Generación del manuscrito para artículo de datos usando el IPT

El IPT en el cual ha sido publicado el conjunto de datos permite generar un manuscrito RTF que describe al conjunto de datos. El enlace al conjunto de datos aparecerá en el manuscrito bajo el título "Data published through GBIF". A continuación se describe el paso a paso para generar el manuscrito desde los metadatos del conjunto de datos publicado a través del SiB Colombia.

- En la página principal del recurso publicado a través del IPT, se debe hacer clic sobre el botón RTF para descargar una versión del manuscrito en texto enriquecido que se puede abrir en cualquier procesador de texto (por ej: Word) (Figura 2). 


\section{Escarabajos coprófagos de la cuenca alta y media del río Bita, Vichada} (Colombia)

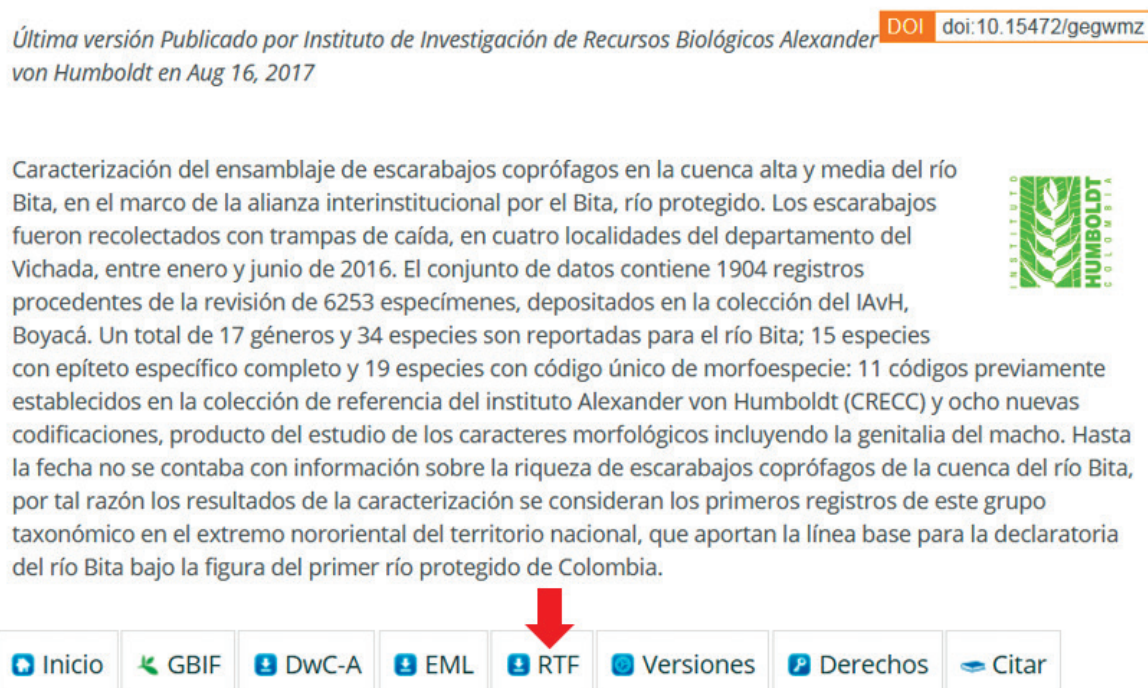

Figura 2. Los metadatos de una conjunto de datos, pueden ser descargados del IPT como archivo RTF, la primera versión del manuscrito para someter a la revista.

- El manuscrito descargado se encuentra en inglés. Los ajustes necesarios de acuerdo a los lineamientos de la revista Biota Colombiana deben ser realizados y la plantilla para artículos de datos se puede descargar aquí.

- Una vez ajustado el manuscrito con los textos adicionales, tablas y figuras, puede ser sometido a evaluación a través del portal en línea de la revista, siguiendo los pasos de registro como usuario. Todo el proceso editorial se desarrolla a través de esa plataforma.

\section{Paso 3}

\section{Ajustes y correcciones del manuscrito para artículos de datos}

Cuando el manuscrito ha sido sometido a evaluación, este se somete a revisión por pares evaluadores de acuerdo a los lineamientos establecidos por la revista para evaluadores de artículos de datos.

Después de evaluado, y en caso de ser aceptado, el manuscrito será devuelto al autor con los comentarios de los revisores y del editor de la revista con el objetivo de realizar las modificaciones antes de publicar. Como autor, deberá realizar todas las correcciones o adiciones recomendadas directamente en los metadatos del IPT y no en el manuscrito del artículo de datos. De esta forma se mejoran también los metadatos del conjunto de datos sometido.

Una vez se hayan mejorado los metadatos en el IPT, se debe actualizar la publicación del recurso para que los cambios se vean reflejados. En la página principal del recurso publicado a través del IPT, ubique el botón RTF y haga clic sobre el mismo para descargar una versión mejorada del manuscrito en texto enriquecido que puede abrir nuevamente en cualquier procesador de texto (por ej. Word).

Después de la re-inserción manual de los textos adicionales y asegurarse que esta versión cumple con los requerimientos de la revista, el manuscrito debe ser enviado nuevamente a la revista.

\section{Secciones de un artículo de datos}

A diferencia de los otros tipos de manuscritos que pueden ser sometidos a la revista Biota Colombiana, los artículos de datos incluyen las secciones estipuladas en la Tabla 1. 
Tabla 1. Estructura del artículo de datos y su correspondencia con los elementos del GMP incorporados en el IPT.

\section{Correspondencia con los elementos del IPT}

Nombre de la sección

\section{Título}

Autores

Afiliaciones

Autores de contacto
Derivado del elemento Título. Centrado sin punto al final.

Derivado de los elementos Creador del recurso, Proveedor de los metadatos y Partes asociadas. De estos elementos, se deriva la combinación nombre y apellido separados por comas. Las afiliaciones de los autores se indican con números $(1,2,3 \ldots)$ al final de cada apellido como superíndice. Centrado.

Derivado de los elementos Creador del recurso, Proveedor de los metadatos y Partes asociadas. De estos elementos, la combinación de organización, dirección, código postal, ciudad, país y correo electrónico, constituyen la afiliación. Si dos o más autores comparten la misma afiliación, se indica con el mismo número.

Derivado de los elementos Creador del recurso y Proveedor de los metadatos. De estos elementos, se deriva la combinación nombre, apellido y correo electrónico. Los correos electrónicos son escritos en paréntesis. Si hay más de un autor como contacto, estos van separados por comas. Si el Creador del recurso y Proveedor de los metadatos es el mismo autor, el Creador del recurso se refleja como el autor de contacto. Texto centrado.

Insertadas manualmente por el Editor Asistente de la revista para indicar las fechas de presentación original del manuscrito, revisión, aceptación y publicación como un artículos de datos en Biota Colombiana.

Fechas de recibido, revisado, aceptado y publicado

\section{Resumen}

Derivado del elemento descripción. El resumen debe incluirse tanto en español o portugués como inglés.

Derivadas del elemento palabras claves. Las palabras van separadas por comas. Las palabras deben estar escritas tanto en español o portugués como inglés.

Palabras Clave

Introducción No se deriva de ningún elemento del GMP y debe ser adicionada manualmente.

Cobertura taxonómica

Derivada de los elementos de la sección cobertura taxonómica: descripción, nombre científico, nombre común y categoría.

Cobertura geográfica

Derivada de los elementos de la sección cobertura geográfica: descripción, latitud mínima, latitud máxima, longitud mínima, longitud máxima.

Cobertura temporal

Derivada de los elementos de la sección cobertura temporal: fecha inicial y fecha final.

Derivada de los elementos de la sección datos del proyecto: título, persona del proyecto, fuentes de financiación, descripción del área de estudio y descripción del diseño.

Descripción del proyecto

Derivada de los elementos de la sección datos de la colección: nombre de la colección,

Descripción de la colección biológica

identificador de la colección, identificador de la colección parental, método de preservación de los especímenes y unidades curatoriales.

Derivado de los elementos de la sección métodos de muestreo: área de estudio, descripción del muestreo, control de calidad, descripción del paso metodológico.

\section{Materiales y métodos}

\section{Resultados}

$--$

Derivado de los elementos de la sección enlaces externos entre otros: nombre, conjunto de caracteres, URL del archivo, formato del archivo, versión del formato del archivo, fecha de publicación, idioma, derechos de propiedad intelectual. Puede adicionar manualmente una descripción adicional de los datos como texto, figuras y tablas.

Información adicional

Derivado del elemento información adicional.

Discusión

No se deriva de ningún elemento del GMP y debe ser adicionada manualmente, enfocada en el potencial de uso de los datos en investigación, educación o toma de decisiones.

Agradecimientos

Referencias

No se deriva de ningún elemento del GMP y debe ser adicionada manualmente.

Derivado del elemento referencia en bibliografía. 


\section{Guidelines for authors}

\section{(http:// revistas.humboldt.org.co/index.php/biota)}

\section{Submitting a manuscript}

Submitting a manuscript implies the explicit statement by the author(s) that the paper has not been published before, nor accepted for publication in another journal or other means of scientific diffusion. Contributions are entire responsibility of the author(s) and not the Research Institute of Biological Resources Alexander von Humboldt, or the journal and their editors.

Papers can be written in Spanish, English or Portuguese, and should not exceed the maximum length of 40 pages (with paragraph lines spaced at 1.5) including tables, figures and appendices. Of particular interest for this journal are descriptions of new species for science, new geographic records, thematic or regional species lists, inventories, databases related to biodiversity, biological collections and sampling reports.

Biota Colombiana receives scientific research articles, as well as notes, reviews, bibliographic novelties and data papers.

Manuscripts must be submitted through the online platform of the journal (http://revistas.humboldt. org.co/index.php/biota) as a registered user. The complete editorial process is managed in this platform.

\section{Evaluation}

Submitted manuscripts will be reviewed by at least two qualified scientific peers. Results of the peer revisions may include any of the following: a) accepted (in this case it is assumed that no change, omission or addition to the article is required and may be published as presented.); b) conditional acceptance (the article is accepted and recommended for publication only if indicated corrections are made; corrections may be minor and a second evaluation is not necessary or major and a second evaluation is necessary); and c) rejected (reviewer considers that the contents and/or form of the paper are not in accordance with requirements of publication standards of Biota Colombiana). For a manuscript to continue its editorial process, it must have been accepted by at least two reviewers.

\section{Preparation of Manuscript}

Any word-processor program may be used to write the text of the manuscript (Word is recommended). Lists or any other type of table must be presented in spreadsheets (Excel is recommended). To submit a manuscript, a cover letter that clearly indicates the following must be sent:

1. Full names, institutional filiations, and e-mail addresses of all authors. (Please note that email addresses are essential to direct communication)

2. Complete title of the article

3. Names, sizes and types of files provided.

4. Concise and clear sustentation of why the presented manuscript is in concordance with the type of articles published in the journal. Such explanation must not surpass a maximum of three lines.

5. List of the names and e-mail addresses of at least four peers who are qualified to review the manuscript.

\footnotetext{
**For information regarding the preparation of data papers, continue to "Details for Data Papers-> Preparation of Data Paper"
} 


\section{Use of Language}

- Manuscripts that are sent to Biota Colombiana have as a minimum requirement for consideration the appropriate use of language in writing, regardless if they are presented in Spanish, Portuguese or English.

- The style should follow the common formalities of scientific writing and be clear, concise and cohesive.

- The use of guides for correct spelling, grammar and style is recommended.

\section{Format}

- Texts must follow the format of standard letter size paper, with $2.5 \mathrm{~cm}$ margins on all sides, 1.5-spaced and left-aligned paragraphs (including title and bibliography).

- All pages must be numbered in the lower right corner.

- Font must be Times New Roman or Arial, size 12, in all parts of the text, except tables (size 10). Manuscript must not exceed a maximum length of 40 pages, including tables, figures and appendices. Avoid the use of bold or underlined font.

- Scientific names of genera, species and subspecies must be in italics, as well as Latin technical terms (i.e sensu, et al.). Avoid underlining any word or title. Do not use footnotes.

- For abbreviations and the metric system, use the standards of the International System of Units (SI). Leave a space between the numeric value and the measure unit (p.e. $16 \mathrm{~km}, 23{ }^{\circ} \mathrm{C}$ ). For relative measures such as $\mathrm{m} / \mathrm{sec}$, use $\mathrm{m} . \mathrm{sec}-1$.

- Write the numbers between one to ten in letters except when it precedes a measure unit (p.e. $9 \mathrm{~cm}$ ) or is used as a marker (p.e. lot 2, sample 7). Numbers greater than ten must be written in Arabic numerals. If in the paragraph both numbers lesser than ten and greater than ten appear, all should be written in Arabic numerals.

- Thousands, millions, etc. should not be separated by commas nor periods (p. e. 54000). Use periods to separate decimals (p. e. 3.1416). In Spanish, use commas to separate decimals (p. e. 3,1416). Hours should be represented in military time from 0:00 to $24: 00$.

- Years should be written without commas or periods (p. e. 1996-1998). In English months and days of the week are capitalized (January, July, Saturday, Monday), whereas in Spanish the first letter of months and days of the week are written with lower-case letters (enero, julio, sábado, lunes).

- Cardinal points (north, south, east and west) must be written in lower case letters, and only be capitalized if used in abbreviation N, S, E, W (O in Spanish) or as part of a proper name ( $p$. e. North Carolina). Correct use of coordinates is: $02^{\circ} 37^{\prime} 53^{\prime \prime} \mathrm{N}-56^{\circ} 28^{\prime} 53^{\prime \prime} \mathrm{O}$. Altitude should be expressed as $1180 \mathrm{~m}$ a.s.l. and $1180 \mathrm{~m}$ s.n.m. in Spanish.

- All abbreviations must be explained the first time they are used.

- When citing in-text references, follow APA citation norms (American Psychological Association Publications Manual, Sixth Edition). Include last names of authors if there are only one or two authors, or the first author followed by et al. (in italics) if there are three or more authors. In the case of two authors, last names must be separated by "\&" (p. e. Cochran \& Goin, 1970). In Spanish, last names of two authors must be separated by " $y$ ". If many references are cited, they must be ordered chronologically and separated by semicolons (p. e. Rojas, 1978; Bailey et al., 1983; Sephton, 2001, 2001). Notice that a comma must be inserted after the authors and before the year (Acevedo, 2009).

- Taxonomic references must not be included in the final list of Literature cited, but they must appear in the text of the manuscript.

- Refer to all figures (graphs, diagrams, illustrations, photos) and tables without abbreviation (p. e. Figure 3, Table 1). All figures and tables should have uniformity in font and letter size. 
- Figures must be clear and have a good quality. Unnecessary complexities (such as 3D effects, frames, etc.) should be avoided. If possible, only use solid colors instead of textures. Letters, numbers or symbols must be in legible sizes.

- All figures must be inserted in the text of the manuscript and sent in a separate file in high quality in the step of "Charge complementary files". For photos and digital figures, files must be in tiff, jpg or png format in a resolution not lower than $300 \mathrm{dpi}$.

- Tables and appendices must have a simple and uniform structure. Footnotes in tables must be as superscript letters. Avoid extensive tables with too much information and dividing lines.

\section{Parts of the Manuscript}

** For information about the parts of data papers, continue to "Details for Data Papers -> Parts of Data Paper".

- Submitted manuscripts must contain the following sections: title, abstract and keywords in English and Spanish, Introduction, Materials and methods, Results, Discussion, Conclusions (optional), Acknowledgements (optional) and Literature cited. At the end of the manuscript, include a list with the tables, figures and appendices.

- Sections subtitles must be written in bold, with only the first letter capitalized. If sections have subtitles, they must be written in bold in the first line of the paragraph, separated by a period from the beginning of the paragraph.

Title: concise and explanatory, must clearly communicate what will be found in the article.

Abstract: a summary of the article, with a maximum length of 200 words. It must include the objectives, methods, results and major conclusions of the study. If the article has a novel or extraordinary finding, it must be highlighted in this section. Abstracts must be written in two languages: Spanish or Portuguese, and English.
Keywords: up to five keywords. They must be complementary to the title (not repeated) and written in Spanish or Portuguese, and English. Words must be separated by periods and presented in alphabetical order. The use of thesaurus to find appropriate synonyms is recommended.

Introduction: presentation of the topic, with enough context to support the rest of the article. The main purpose or objective of the study must be made explicit in this section.

Materials and methods: a detailed description of the procedure, with materials, location, dates, statistics, etc. This description must be sufficiently detailed so that other researchers may replicate the study. If a novel procedure is used, it must be thoroughly explained.

Results: presents major findings in an organized and appropriate manner. Avoids the use of excessively long tables.

Discussion: most relevant, troublesome or novel points of the study are highlighted, and major results are explained in relation to the importance of the study and contributions to its field.

Conclusions: final reflections about the study, with a clear relation to its purpose and objectives, frequently pointing towards future actions and research.

Acknowledgements: Straightfoward and short paragraph between text of manuscript and Literature cited. Mention funding or support of the project. Avoid titles such as Dr., Lic., etc.

Literature cited: Follow APA citation norms (American Psychological Association Publications Manual, Sixth Edition). List of references must only include those that are cited within the text. Order the references in alphabetical order, and chronologically in the case of a sole author. If there are many references of a same author (s) in the same year, add letters a, b, c, etc. to the year. Do not abbreviate names of journals. Include all authors. This section must be at the end of the manuscript. 


\section{Citation examples}

\section{Article in journals:}

Antonelli, A., Nylander, J. A., Persson, C. \& Sanmartín, I. (2009). Tracing the impact of the Andean uplift on Neotropical plant evolution. Proceedings of the National Academy of Sciences, 106(24): 9749-9754.

\section{Books:}

Gutiérrez, F. P. (2010). Los recursos hidrobiológicos $y$ pesqueros en Colombia. Bogotá: Instituto de Investigación de Recursos Biológicos Alexander von Humboldt. 118 pp.

\section{Thesis:}

Cipamocha, C. A. (2002). Caracterización de especies y evaluación trófica de la subienda de peces en el raudal Chorro de Córdoba, bajo río Caquetá, Amazonas, Colombia. (Thesis). Bogotá D. C.: Universidad Nacional de Colombia, Facultad de Ciencias, Departamento de Biología.

\section{Technical reports:}

Andrade, G. I. (2010). Gestión del conocimiento para la gestión de la biodiversidad: bases conceptuales y propuesta programática para la reingeniería del Instituto Humboldt. (Technical report). Instituto de Investigación de Recursos Biológicos Alexander von Humboldt. Bogotá D. C., 80 pp.

\section{Book or report chapter:}

Fernández F., Palacio, E. E. \& MacKay, W. P. (1996). Introducción al estudio de las hormigas (Hymenoptera: Formicidae) de Colombia. In Amat, G. D., Andrade, G. \& Fernández, F. (Eds.). Insectos de Colombia. Estudios Escogidos. Pp: 349-412. Bogotá: Academia Colombiana de Ciencias Exactas, Físicas y Naturales \& Centro Editorial Javeriano.

\section{Congress, symposium or workshop summary:}

Señaris, J. C. (2001). Distribución geográfica y utilización del hábitat de las ranas de cristal (Anura; Centrolenidae) en Venezuela. Presented in Programa y Libro de Resúmenes del IV Congreso Venezolano de Ecología, Mérida, Venezuela. p. 124.

\section{Law or decree:}

Congreso de Colombia. (February 8th 1994) Ley General de Educación. [Ley 115 de 1994]. DO: 41.214.

\section{Web pages:}

Must be clearly included in the text of the manuscript, but not be included in Literature cited section.

\section{Details for Data Papers}

A Data Paper is a type of scientific publication that was designed to stimulate the publication of biodiversity data. Data Papers give academic and professional acknowledgement to those who intervene, in one way or another, in the management of information about biodiversity, as well as highlight the existence and importance of data sets to the rest of the scientific community.

As its name suggests, a Data Paper describes a primary data set. Although a Data Paper is not, strictly speaking, a scientific investigation, it must contain relevant information about the data set (objectives, methods for data collection, funding, taxonomic and geographic coverage, etc.), along with its value and utility (basic or applied) for the scientific community (Chavan \& Penev, 2011) ${ }^{1}$. The great advantage and novelty of this type of manuscript is that it is linked to the data set through a stable and trustworthy repository, the IPT (Integrated Publishing Toolkit). Also, the data set is supported by metadata also available through the IPT and linked to the Data Paper.

A Data Paper must be submitted only when the linked data are primary and original data that have a temporal and methodological restriction and are available in data aggregators such as $\underline{\mathrm{SiB} \text { Colombia }}$ and GBIF. Data must be able to follow the Darwin Core $(\mathrm{DwC})$ standard. Examples of such data sets include:

- Project observations

- Biological collections

- Species lists

\footnotetext{
${ }^{1}$ Chavan, V. y Penev, L. (2011). The data paper: The mechanism to incentivize data publishing in biodiversity science. BMC Bioinformatics 2011, 12(Sup. 15): S2
} 
- Genomic data

- Samples

- Inventories

- Databases

- Functional traits

Data sets that do not comply with the characteristics mentioned above will not be accepted for publication as a Data Paper. Such is the case of compilations of biological records that come from secondary sources (p. e. from published literature).

\section{Preparation of Data Paper (publication of data and creation of manuscript)}

Since the purpose of a Data Paper is to describe all available data resources regarding biodiversity, it must always be linked to the data set it describes through an URL or DOI.

Information about how to generate and submit a manuscript in order for it to be considered as a Data Paper by using the tools and publication model of SiB Colombia is found below. It must be noted, however, that Biota Colombiana also accepts Data Papers that link to data sets published in other known platforms as long as it is linked to a trustworthy repository and has an IPT link. The parts of a Data Paper manuscript are described in Table 1.
As other types of manuscripts that are submitted to the journal, Data Papers will be reviewed by peers and must comply with the same format specifications, citation norms and use of language. Similarly, Data Papers must also be presented with a cover letter, as mentioned in the present Guidelines for authors. Have in mind that as soon as the manuscript is submitted and under evaluation, described data must be available in a public online repository with an adequate license of use and attribution.

\section{Step 1}

\section{Data publication in SiB Colombia}

$\mathrm{SiB}$ Colombia uses a publication model pased on the IPT as its working tool. Using the IPT, the first version of the manuscript may be generated in rich text format (RTF), based on its associated metadata. This tool is available as long as the data set has been indexed by $\mathrm{SiB}$ Colombia and sufficient metadata are linked (more information on publication process of $\mathrm{SiB}$ Colombia may be consulted in https://www.sibcolombia.net/).

A. Registration of organisation. To publish through SiB Colombia, your organisation must be registered as a publishing partner. Consult this link to find already registered organisations. If your organisation is not registered, adding an organisation is easy through the Registration Format.

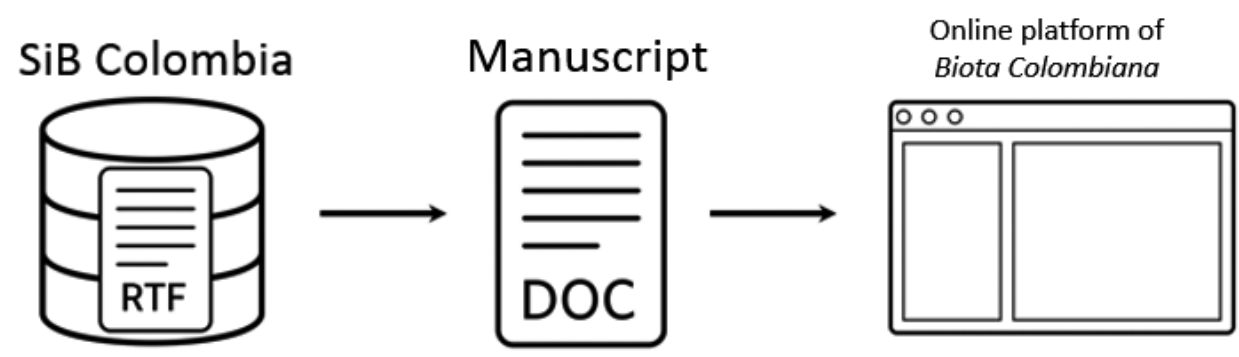

Figura 1. General process to submit a Data Paper from SiB Colombia to the journal Biota Colombiana. 
B. Data standardization. Data must be structured in a table using the Darwin Core $(\mathrm{DwC})$ standard. Download respective template that is appropriate for type of data or generate your template.

C. Data quality. Data quality must be verified and improved using available tools to identify and correct possible geographical, taxonomic or format errors, among others.

D. Online upload of data. IPT is a tool that shares different types of biodiversity data as long as data is structured using DwC standard. To upload data to the IPT, you must have an existing user account in the available IPT of SiB Colombia. If you do not have an account, you may contact the SiB Colombia Coordinating Team (EC-SiB) and request an account to the email address sib@humboldt.org.co.

E. Data mapping. Once the data set is uploaded, verify that it follows DwC elements. For more information, consult the IPT User Manual or contact EC-SiB.

F. Creation of metadata. Metadata structure is similar to that of a traditional research article. In this way, metadata has the same general structure of a Data Paper and thus facilitate the generation of the manuscript. In the metadata section of the IPT, all information that broadens the context of data must be included. There is a total of 12 sections to add information as metadata to describe the data set. For more information, consult the IPT User Manual or contact EC-SiB.

G. Publish resource and notify EC-SiB. Once all previous instructions are completed, IPT will be activate the "Publish" option. Click on the button and send an e-mail to sib@humboldt.org.co in order to notify EC-SiB about your publication. The e-mail must have as subject "Published resource" and include:

- Name

- Name of organisation

- Name of published resource
- URL of general view of resource after publication

Now data are indexed by SiB Colombia and GBIF, and have a digital object identifier, DOI.

Step 2

Creation of manuscript for submission as Data Paper through IPT

The IPT used for publishing the data set generates a RTF manuscript that describes the data set. The link to the data set in the manuscript appears under the title "Data published through GBIF". Here you will find step by step information about how to generate a manuscript based on the data set metadata published in SiB Colombia.

- On the resource homepage published in IPT, click on the RTF button to download the first version of the manuscript in rich text format, which may be opened in any text processor (p. e. Word) (Figure 2).

- Downloaded manuscript is in English. Necessary corrections to follow Biota Colombiana guidelines must be completed on the template. Data Papers template may be downloaded here.

- Once the manuscript is adjusted with additional text, tables and figures, it may be submitted to the journal Biota Colombiana through its online platform, following steps of registry as a user. The complete editorial process is developed through this platform.

\section{Step 3}

Adjustments and corrections of manuscript for data paper

When a manuscript is submitted as a Data Paper, it will go through the same peer review process as other articles of the journal, with specifications for Data Paper evaluation.

After evaluation, and if the manuscript is accepted, it will be returned to the author with the comments of reviewers and the journal's Editor so that respective modifications may be incorporated. 


\section{Escarabajos coprófagos de la cuenca alta y media del río Bita, Vichada (Colombia)}

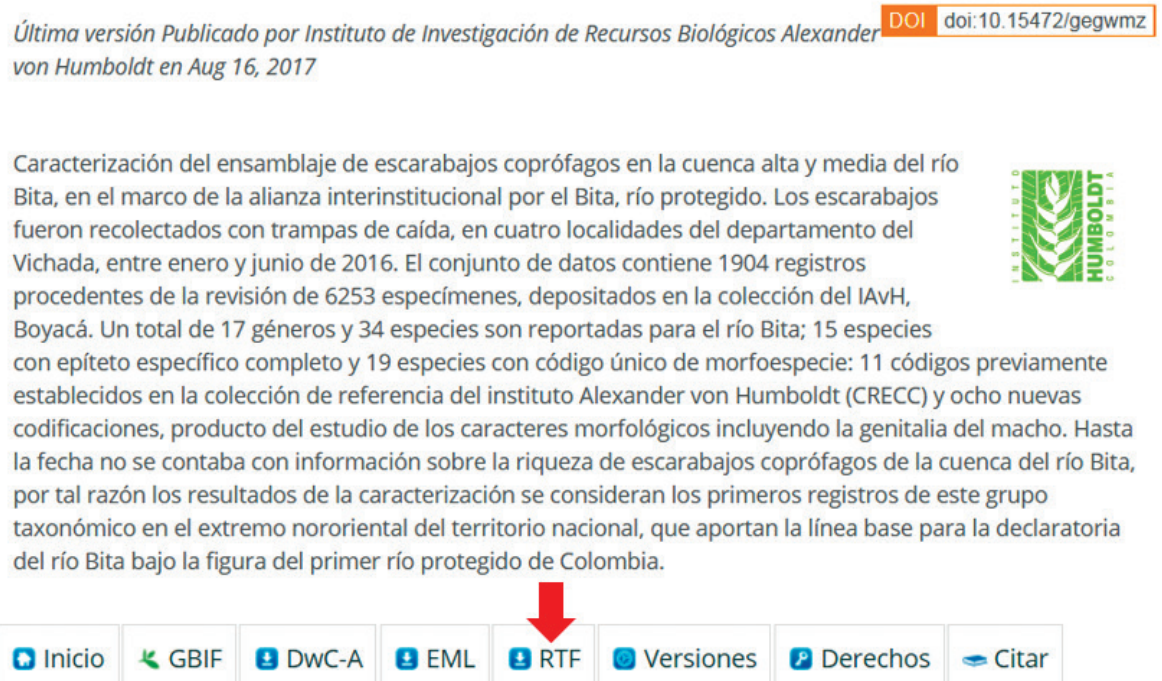

Figure 2. Metadata of a data set may be downloaded from the IPT as a RTF file, giving the first version of the manuscript that will be submitted to the journal.

As the author, you should do all corrections or modifications directly on the IPT metadata and not on the manuscript. In this way, the metadata of the linked data set are also improved by the peer review and editorial comments.

Once metadata in the IPT are improved, the resource publication must be updated so the changes are reflected. On the resource homepage of the published resource, click on the RTF button to download the improved version of the manuscript in rich text format that may be opened in any type of text processor (p. e. Word).
After the manual changes of additional text, figures and tables, and the corroboration that the manuscript follows all of the journal's requirements, it must be sent again through the online platform of Biota Colombiana.

\section{Parts of a Data Paper}

Data Papers differ from other articles that are published in the journal Biota Colombiana in the sections it should include and are mentioned in Table 1. 
Table 1. Structure of a Data Paper and correspondence with GMP elements of IPT.

Name of section

\section{Title}

Authors

Affiliations

\section{Correspondence with IPT elements}

Derived from the element Title. Centered and without period at the end.

Derived from the elements Resource creators, Metadata providers and Associated Parties. From the elements, the combination of name and last name, separated by a coma, is created. Author affiliations are indicated with numbers $(1,2,3 \ldots)$ at the end of each last name with a superscript. Centered.

Derived from the elements Resource creators, Metadata providers and Associated Parties. From these elements, the combination of organisation, address, postal code, city, country and email address constitute the complete affiliation. If one or more authors share the same affiliation, it is represented with the same number.

Derived from the elements Resource creators and Metadata providers. From the elements, the combination of name, last name, and email address is created. Email addresses are inside parentheses. If there is more than one author as contact, authors are separated by comas. If the Resource creator and Metadata provider is the same author, the Resource creator is assumed to be the contact. Text is centered.

\section{Dates of received, revised, accepted and published}

Manually incorporated by the editorial assistant of the journal to indicate respective dates of when the manuscript was received, revised, accepted and published as a Data Paper in Biota Colombiana.

\begin{tabular}{|c|c|}
\hline Abstract & $\begin{array}{l}\text { Derived from the element description. Abstract must be included in Spanish or } \\
\text { Portuguese, and English. }\end{array}$ \\
\hline Keywords & $\begin{array}{l}\text { Derived from the element keywords. Words are separated by comas. Keywords must be } \\
\text { written in Spanish or Portuguese, and English. }\end{array}$ \\
\hline Introduction & Not derived and must be added by the authors manually. \\
\hline Taxonomic coverage & $\begin{array}{l}\text { Derived from section of taxonomic coverage: description, scientific names, common names } \\
\text { and category. }\end{array}$ \\
\hline Geographic coverage & $\begin{array}{l}\text { Derived from section of geographic coverage: description, minimum latitude, maximum } \\
\text { latitude, minimum longitude, maximum longitude. }\end{array}$ \\
\hline Temporal coverage & Derived from section of temporal coverage: description, start date, end date. \\
\hline Project description & $\begin{array}{l}\text { Derived from section of project data: title, project personnel, funding, study area description, } \\
\text { design description. }\end{array}$ \\
\hline Collection data & $\begin{array}{l}\text { Derived from section of collection data: name of collection, collection identifier, parental } \\
\text { collection identifier, specimen preservation methods, curatorial units. }\end{array}$ \\
\hline Materials and methods & $\begin{array}{l}\text { Derived from section of sampling methods: study extent, study description, quality control, } \\
\text { step description. }\end{array}$ \\
\hline Results & --- \\
\hline Data description & $\begin{array}{l}\text { Derived from external links, among others: name, file URL, file format, version of file format, } \\
\text { publication date, language, copyright. An additional description of data such as text, figures } \\
\text { and tables may be added. }\end{array}$ \\
\hline Additional information & Derived from element of additional information. \\
\hline Discussion & Not derived and must be added by the authors manually. \\
\hline Acknowledgements & Not derived and must be added by the authors manually. \\
\hline Literature cited & Derived from element of citations. \\
\hline
\end{tabular}




\section{Biota Colombiana}

Volumen 19 (Sup. 1) - Colombia Bio y otras novedades científicas

Una publicación del / A publication of: Instituto de Investigación de Recursos Biológicos Alexander von Humboldt

En asocio con / In collaboration with:

Instituto de Ciencias Naturales de la Universidad Nacional de Colombia

Instituto de Investigaciones Marinas y Costeras - Invemar

Missouri Botanical Garden

\section{TABLA DE CONTENIDO/ TABLE OF CONTENTS}

Editorial

Una nueva especie de barniz de pasto Elaeagia (Rubiaceae), de la cordillera Oriental de Colombia. A new species of Elaeagia (Rubiaceae) from the cordillera Oriental of Colombia. Humberto Mendoza-Cifuentes y José Aguilar-Cano

Una nueva especie de Allomaieta (Melastomataceae - Cyphostyleae) del piedemonte amazónico de los Andes de Colombia. A new species of Allomaieta (Melastomataceae-Cyphostyleae) from the Amazonian foothills of the Colombian Andes. Humberto Mendoza-Cifuentes

Dos nuevas especies de Miconia (Melastomataceae) del piedemonte oriental de la cordillera Central de Antioquia, Colombia. Two new species of Miconia (Melastomataceae) from the eastern foothills of the Cordillera Central of Antioquia, Colombia. Humberto Mendoza-Cifuentes, Julián Aguirre-Santoro y Álvaro Idárraga

Dos nuevas especies de árboles molinillo (Magnolia: Magnoliaceae) de la serranía de los Yariguíes, departamento de Santander, Colombia. Two new species of "molinillo" tree (Magnolia: Magnoliaceae) from Serranía de los Yariguíes, Santander, Colombia. José Aguilar-Cano, Humberto Mendoza-Cifuentes y Melisa Ayala-Joya

Catálogo de la flora de los Parques Nacionales de Colombia: Parque Nacional Natural El Tuparro. Catalogue of the flora of the National Natural Parks of Colombia: El Tuparro National Natural Park. Humberto Mendoza-Cifuentes y Mireya P. Córdoba-Sánchez .

Primer registro del efemeróptero Oligoneuria (Oligoneurioides) amazonica (Demoulin, 1955) (Insecta: Ephemeroptera, Oligoneuriidae) para Colombia. First record of Mayfly Oligoneuria (Oligoneurioides) amazonica (Demoulin, 1955) (Insecta: Ephemeroptera, Oligoneuriidae) from Colombia. Cristian E. Granados-Martínez, Carlos A. Lasso y Juan M. Fuentes-Reinés .....

Variaciones morfológicas y algunas notas bioecológicas del cangrejo de agua dulce Neostrengeria charalensis Campos y Rodríguez, 1985 (Decapoda: Pseudothelphusidae), en ambientes exo y endocársticos de los Andes colombianos. Morphological variations and some bioecological notes of the freshwater crab Neostrengeria charalensis Campos \& Rodríguez, 1985 (Decapoda: Pseudothelphusidae), in exo and endocárstic environments of the Colombian Andes. Martha R. Campos, Ada Acevedo, Carlos A. Lasso y Jesús Fernández-Auderset

Ectoparásitos (Argulidae, Cymothoidae, Corallanidae) en rayas de agua dulce (Potamotrygonidae) de la Orinoquia colombiana. Ectoparasites (Argulidae, Cymothoidae, Corallanidae) in freshwater rays (Potamotrygonidae) of the Colombian Orinoquia. Carlos A. Lasso, Martha R. Campos, Mónica A. Morales-Betancourt y David Castro .....

Trichomycterus rosablanca (Siluriformes, Trichomycteridae) a new species of hipogean catfish from the Colombian Andes. Trichomycterus rosablanca (Siluriformes, Trichomycteridae) una especie nueva de bagre hipogeo de los Andes colombianos. Lina M. Mesa S., Carlos A. Lasso, Luz E. Ochoa y Carlos DoNascimiento

A new species of cave catfish, genus Trichomycterus (Siluriformes: Trichomycteridae), from the Magdalena River system, Cordillera Oriental, Colombia. Una nueva especie de bagre de caverna, género Trichomycterus (Siluriformes: Trichomycteridae), del sistema río Magdalena, cordillera Oriental, Colombia. César A. Castellanos-Morales

Una nueva rana de huesos verdes del género Scinax (Anura: Hylidae) asociada a los bosques subandinos de la cuenca del río Magdalena, Colombia. A new frog with green bones of the genus Scinax (Anura: Hylidae), associated with the sub-Andean forests of the Magdalena River basin, Colombia. Andrés R. Acosta-Galvis

Una nueva rana nodriza (Anura: Dendrobatidae) de los bosques de niebla asociados a la cuenca del Orinoco de Colombia. A new nurse frog (Anura: Dendrobatidae) from the cloud forests of the Orinoco basin of Colombia. Andrés R. Acosta-Galvis y Adrián Pinzón

\section{Notas}

Nuevos registros de plantas acuáticas para la región Guayana y notas sobre las islas flotantes en el río Guaviare, Guainía, Colombia. New records of aquatic plants from the Guayana region in Colombia, with notes on floating islands in the Guaviare River, Guainía. Anabel Rial

Primer registro del hemíptero Strudivelia cinctipes Champion, 1898 (Hemiptera: Veliidae) para ambientes cavernícolas de Colombia. First record of the hemiptera Strudivelia cinctipes Champion, 1898 (Hemiptera: Veliidae) for cave environments in Colombia. Hernán Aristizábal-García, Natalia Herreño-Castellanos y Carlos A. Lasso 